\title{
Ionospheric control of the magnetosphere: conductance
}

\author{
A. J. Ridley, T. I. Gombosi, and D. L. DeZeeuw \\ The University of Michigan, Ann Arbor, USA
}

Received: 13 September 2002 - Revised: 27 May 2003 - Accepted: 27 June 2003 - Published: 1 January 2004

\begin{abstract}
It is well known that the ionosphere plays a role in determining the global state of the magnetosphere. The ionosphere allows magnetospheric currents to close, thereby allowing magnetospheric convection to occur. The amount of current which can be carried through the ionosphere is mainly determined by the ionospheric conductivity. This paper starts to quantify the nonlinear relationship between the ionospheric conductivity and the global state of the magnetosphere. It is found that the steady-state magnetosphere acts neither as a current nor as a voltage generator; a uniform Hall conductance can influence the potential pattern at low latitudes, but not at high latitude; the EUV generated conductance forces the currents to close in the sunlight, while the potential is large on the nightside; the solar generated Hall conductances cause a large asymmetry between the dawn and dusk potential, which effects the pressure distribution in the magnetosphere; a uniform polar cap potential removes some of this asymmetry; the potential difference between solar minimum and maximum is $\sim 11 \%$; and the auroral precipitation can be related to the local field-aligned current through an exponential function.
\end{abstract}

Key words. Ionosphere (ionosphere-magnetosphere interactions; modelling and forecasting; polar ionosphere)

\section{Introduction}

This study is one in a series of three which examines the influence of the thermosphere and ionosphere on the global state of the magnetosphere. The present study examines the influence of the ionospheric conductance on the magnetospheric configuration. The two other studies examine how the thermospheric neutral winds effect the ionospheric electric field and magnetospheric convection (Ridley et al., 2003), and the influence of ionospheric outflow on the time-dependent magnetospheric configuration (Ridley et al., 2002a).

The closing of the magnetospheric currents through the ionosphere is one of the most important aspects of the magnetospheric system. The magnetospheric currents flow into

Correspondence to: A. J. Ridley (ridley@umich.edu) the ionosphere through the Birkeland currents, which are described by Iijima and Potemra (1976). Region 1 currents, which flow at high latitudes, are considered a direct result of the solar wind and the IMF driving the magnetosphere. Region 2 currents are equatorward of the region 1 currents and are caused by pressure gradients in the inner magnetosphere. These currents close through the conductive ionosphere.

The ionospheric perpendicular conductivity is a quantification of the amount of resistance which the ions and electrons encounter when moving through the neutral atmosphere. Above $\sim 150 \mathrm{~km}$, there are very few collisions between ions, electrons, and neutrals. Because there are few collisions, the perpendicular conductivity is very low in this region. Below $150 \mathrm{~km}$, and above $\sim 100 \mathrm{~km}$, the ions collide with the neutrals often, while electrons still have very few collisions with the neutrals. The ion-neutral collisions cause the ions to turn from the $\mathbf{E} \times \mathbf{B}$ direction to the $\boldsymbol{E}$ direction, while the electrons continue to $\mathbf{E} \times \mathbf{B}$ drift. This diverted ion motion creates the Pedersen current, while the electrons create the Hall current. The relative amounts of current which are driven by a given electric field are specified by the Hall and Pedersen conductances (e.g. Song et al., 2001).

The importance of the ionospheric conductance caused by solar radiation and particle precipitation is indicated by the number of existing conductance models. Most heightintegrated solar driven conductance models are solar zenith angle dependent, because extreme ultraviolet (EUV) solar radiation is absorbed along a line between the given point and the Sun (e.g. Green et al., 1964). The Sun's EUV spectrum is absorbed in the upper atmosphere, creating photoelectrons which give rise to ionospheric conductivities (e.g. Torr et al., 1979). Brekke and Moen (1993) review a number of solar driven ionospheric conductance models. Lilensten et al. (1996) show theoretical calculations of the solar derived auroral zone latitude conductances and compare those with the emprical relationship derived by Senior (1991).

Auroral electron and ion precipitation causes ionization throughout the thermosphere (e.g. Frahm et al., 1997; Galand et al., 2001). This implies that the precipitation can be related to the Hall and Pedersen conductances (Robinson et al., 1987). A number of emprical models of electron precipitation exist. These models relate the electron particle 
precipitation to various indices, such as $A E$ and $K_{p}$ (Spiro et al., 1982; Hardy et al., 1985; Ridley et al., 1999), or degrees of stretching in the magnetotail (Solirelis and Newell, 2000). The model of Fuller-Rowell and Evans (1987) relates the ionospheric Hall and Pedersen conductance to the hemispheric power index, and was created using electron precipitation data as well. Galand et al. (2001) derive conductances from ion precipitation. The formulations given by Ahn et al. (1983) and Ahn et al. (1998) relate the Hall and Pedersen conductances to ground-based magnetometer measurements.

Even though many studies are concerned with the conductance itself, very few studies have actually examined the influence of the ionospheric conductivity on the magnetospheric configuration or dynamics. This is very difficult to do with data, since measurements are very sparse in the magnetosphere. In order to conduct studies of this type, one would like to have very similar driving conditions in the solar wind with vastly different ionospheric conductances (e.g. solar maximum versus solar minimum, or summer versus winter, etc.) In addition, the satellites would have to be in approximately the same place, in order to insure that the measurements are coming from the same region of the magnetosphere. Since the combination of events never actually occurs, it is very difficult to observationally determine how the ionospheric conductance truly influences the magnetospheric configuration and dynamics.

We must, therefore, turn to models to attempt to quantify what effects the ionosphere has on the magnetosphere. A number of studies have been conducted using global magnetosphere codes. For example, Fedder and Lyon (1987) showed that the magnetosphere is neither a current nor a voltage generator, using a global magnetohydrodynamic (MHD) code. They did this by varying a constant ionospheric conductance to determine whether the magnetosphere would self-consistently increase the field-aligned currents or not.

Raeder et al. (1996) showed that the ionospheric conductance specification is crucial in determining the temporal history of the magnetospheric dynamics. They showed that by changing the conductance in the ionosphere, they could make the magnetosphere go into either a steady convection state or a storage-unloading state. Raeder et al. (2001) further describes how the ionospheric conductance can determine the timing and strength of the auroral substorms through the use of a limited parametric study. While these two studies are very insightful and show that the conductance has a strong effect on the dynamics of the magnetosphere, the modeled time period is too complex to quantify the cause and effect relationship between the ionospheric and magnetospheric processes.

The study by Siscoe et al. (2002) furthered the earlier work by Hill and Rassbach (1975) by using a global MHD model of the magnetosphere to show that some of the simple analytic relationships which Hill and Rassbach (1975) derived were valid in a global simulation. They derived an analytic relationship between the saturation of the cross polar cap potential and the globally averaged ionospheric conductance.
This study attempts to continue the work started by Fedder and Lyon (1987), by examining simplified steady-state conditions of the magnetosphere, and how these steady-states depend upon the ionospheric conductance. Understanding the steady-state magnetosphere is crucial when attempting to understand the dynamic magnetosphere. Therefore, further studies will examine the magnetospheric dynamics dependence upon the conductance.

\section{Technique}

Goodman (1995) described in great detail the methodology for solving for the inner boundary condition in a global MHD code. Amm (1996) pointed out some differences in the specified conductance formulation given by the Goodman (1995) study. This methodology has been incorporated into the University of Michigan MHD code (Powell et al., 1999; Ridley et al., 2001, 2002). The method can be summarize in the following steps:

1. The field-aligned currents at 3.5 Earth radii $\left(R_{E}\right)$ are computed using $J_{\|}=(\nabla \times \mathbf{B}) \cdot \mathbf{b}$, where $J_{\|}$is the fieldaligned current, $\mathbf{B}$ is the local magnetic field, and $\mathbf{b}$ is the direction of the local magnetic field.

2. These field-aligned currents are mapped down to the ionosphere using the background (i.e. dipolar) magnetic field, and scaled according to the ratio $B_{I} / B_{3.5}$, where $B_{3.5}$ and $B_{I}$ are the strengths of the magnetic field at $3.5 R_{E}$ and the ionosphere (i.e. $120 \mathrm{~km}$ altitude), respectfully.

3. A conductance pattern is generated. This step will be discussed in detail in the rest of the text.

4. The electric potential is solved for using the relationship $j_{R}\left(R_{I}\right)=\left[\nabla_{\perp} \cdot(\Sigma \cdot \nabla \psi)_{\perp}\right]_{R=R_{I}}$, where $\Sigma$ is the ionospheric conductance tensor, and $\psi$ is the potential.

5. The ionospheric potential is mapped to the inner boundary of the simulation (i.e. $2.5 R_{E}$ ), using the background magnetic field.

6. The electric fields and velocities at the inner boundary are calculated using the relationships $\mathbf{E}=-\nabla \psi$ and $\mathbf{V}=\mathbf{E} \times \mathbf{B} / B^{2}$, where $\mathbf{E}$ is the electric field. At this point, the corotation velocity field is added to the ionospheric generated velocity field. The total velocity field is used as an inner boundary condition in the simulation.

The relationship in 4 above can be expanded to (Amm, 1996):

$$
\begin{aligned}
& j_{R}\left(R_{I}, \theta, \psi\right)= \\
& \frac{1}{R_{I}^{2}}\left[\frac{\delta^{2} \Phi}{\delta \theta^{2}} \frac{\Sigma_{0} \Sigma_{P}}{C}+\right. \\
& \frac{\delta^{2} \Phi}{\delta \psi^{2}}\left\{\frac{1}{\sin ^{2} \theta}\left(\Sigma_{P}+\frac{\Sigma_{H}^{2} \sin ^{2} \varepsilon}{C}\right)\right\}+
\end{aligned}
$$




$$
\begin{aligned}
& \frac{\delta \Phi}{\delta \theta}\left\{\frac{\delta}{\delta \theta}\left(\frac{\Sigma_{0} \Sigma_{P}}{C}\right)+\cot \theta \frac{\Sigma_{0} \Sigma_{P}}{C}+\right. \\
& \left.\frac{1}{\sin \theta} \frac{\delta}{\delta \psi}\left(\frac{\Sigma_{0} \Sigma_{H} \cos \varepsilon}{C}\right)\right\}+ \\
& \frac{\delta \Phi}{\delta \psi}\left\{\frac{\delta}{\delta \theta}\left(\frac{\Sigma_{0} \Sigma_{H}(-\cos \varepsilon)}{C \sin \theta}\right)+\right. \\
& \frac{1}{\sin ^{2} \theta} \frac{\delta}{\delta \psi}\left(\Sigma_{P}+\frac{\Sigma_{H}^{2} \sin ^{2} \varepsilon}{C}\right)+ \\
& \left.\left.\frac{\Sigma_{0} \Sigma_{H}(-\cos \varepsilon) \cot \theta}{C \sin \theta}\right\}\right]_{R=R_{I}},
\end{aligned}
$$

where

$C=\Sigma_{0} \cos ^{2} \varepsilon+\Sigma_{P} \sin ^{2} \varepsilon$

$\varepsilon$ is the angle between the radial direction and the magnetic field, $\theta$ is the colatitude, and $\Sigma_{0}, \Sigma_{H}$, and $\Sigma_{P}$ are fieldaligned, Hall, and Pedersen height integrated conductances, respectfully. This equation makes no assumptions about the spatial distribution of conductances, the magnetic field, or the electric potential. Within the MHD code discussed here, $\Sigma_{0}$ is given as a constant value of 1000 mhos.

The electric potential is solved by using a preconditioned gradiant reduction resolution (GMRES) solver, which is robust enough to handle a wide variety of field-aligned current and conductance patterns. The potential in each hemisphere is determined independently, with a boundary condition of zero potential at $5^{\circ}$ latitude lower than the lowest latitude field-aligned current. In the simulations described below, this is at $\sim 60^{\circ}$ (magnetic) latitude. This boundary condition is used primarily to act in place of a shielding region 2 current. Since the MHD code does not produce very strong region 2 currents (see Ridley et al., 2002, for more of a discussion), the region 1 currents would be unshielded and would extend to very low latitudes. Since this is not observed, except in time periods when the region 1 currents are changing and penetration electric fields can exist, this is a relatively good approximation.

\section{Uniform conductances}

In this section, we discuss the configuration of the steadystate magnetosphere under the assumption of a uniform ionospheric conductance. This is a physically unrealistic case, but in some localized regions (the dayside), it may be approximately correct. When this assumption is made, Eq. (1) is simplified to:

$$
\begin{aligned}
& j_{R}\left(R_{I}, \theta, \psi\right)= \\
& \frac{1}{R_{I}^{2}}\left[\frac{\delta^{2} \Phi}{\delta \theta^{2}} \frac{\Sigma_{0} \Sigma_{P}}{C}+\frac{\delta^{2} \Phi}{\delta \psi^{2}}\left\{\frac{1}{\sin ^{2} \theta}\left(\Sigma_{P}+\frac{\Sigma_{H}^{2} \sin ^{2} \varepsilon}{C}\right)\right\}+\right. \\
& \frac{\delta \Phi}{\delta \theta}\left\{\Sigma_{0} \Sigma_{P} \frac{\delta C^{-1}}{\delta \theta}+\cot \theta \frac{\Sigma_{0} \Sigma_{P}}{C}+\right. \\
& \left.\frac{\Sigma_{0} \Sigma_{H}}{\sin \theta} \frac{\delta}{\delta \psi}\left(C^{-1} \cos \varepsilon\right)\right\}+
\end{aligned}
$$

$$
\begin{aligned}
& \frac{\delta \Phi}{\delta \psi}\left\{-\Sigma_{0} \Sigma_{H} \frac{\delta}{\delta \theta}\left(C^{-1} \cos \varepsilon \sin ^{-1} \theta\right)+\right. \\
& \left.\left.\frac{\Sigma_{H}^{2}}{\sin ^{2} \theta} \frac{\delta}{\delta \psi}\left(\frac{\sin ^{2} \varepsilon}{C}\right)+\frac{\Sigma_{0} \Sigma_{H}(-\cos \varepsilon) \cot \theta}{C \sin \theta}\right\}\right]_{R=R_{I}} .
\end{aligned}
$$

Many models (such as MHD models) assume that the ionospheric conductances are globally uniform. We start with the simplest case, of uniform Pedersen conductance and zero Hall conductance and then consider a uniform non-zero Hall conductance.

\subsection{Zero Hall conductance}

The simplest ionospheric conductance pattern which can produce a quasi-realistic magnetospheric configuration is a uniform zero Hall conductance and a uniform non-zero Pedersen conductance. This is a standard ionosphere for many MHD model runs (e.g. Fedder and Lyon, 1987). For steady-state runs the uniform conductances produce very smooth electric potential patterns. In addition, the magnetosphere and ionosphere are symmetric across the noon-midnight meridian and the equator for simulations in which the IMF is only vertical (i.e. $B_{y}=B_{x}=0$ and corotation is neglected. Using these simplifying assumptions, Eq. (3) becomes:

$$
\begin{aligned}
& j_{R}\left(R_{I}, \theta, \psi\right)= \\
& \frac{1}{R_{I}^{2}}\left[\frac{\delta^{2} \Phi}{\delta \theta^{2}} \frac{\Sigma_{0} \Sigma_{P}}{C}+\frac{\delta^{2} \Phi}{\delta \psi^{2}} \frac{\Sigma_{P}}{\sin ^{2} \theta}+\right. \\
& \left.\frac{\delta \Phi}{\delta \theta}\left\{\Sigma_{0} \Sigma_{P} \frac{\delta C^{-1}}{\delta \theta}+\cot \theta \frac{\Sigma_{0} \Sigma_{P}}{C}\right\}\right]_{R=R_{I}} .
\end{aligned}
$$

Several models and studies assume that the field lines are vertical (i.e. $\varepsilon=0$ ). For example, Fukushima (1969) showed that ground magnetometers will measure only the ionospheric Hall current if the magnetic field lines are vertical, and the Hall and Pedersen conductances are uniform. This is because the magnetic perturbation on the ground due to the Pedersen and field-aligned currents will exactly cancel if these assumptions are true. Because of these assumptions, the horizontal component of the ground magnetic perturbation can be easily related to the ionospheric electric field (see, for example, McHenry and Clauer, 1987).

Another example is the assimilative mapping of the ionospheric electrodynamics (AMIE) technique (Richmond and Kamide, 1988). AMIE allows for the horizontal height integrated conductivities to be highly nonuniform, but assumes that the magnetic field is vertical. This is done so that the ground-based magnetic perturbations can be easily related to the ionospheric electric potential.

If we make the same assumption ( $\varepsilon=0$ globally) we simplify Eq. (4) to:

$$
\left.j_{R}(R) I, \theta, \psi\right)=\frac{\Sigma_{P}}{R_{I}^{2}}\left[\frac{\delta^{2} \Phi}{\delta \theta^{2}}+\frac{1}{\sin ^{2} \theta} \frac{\delta^{2} \Phi}{\delta \psi^{2}}+\cot \theta \frac{\delta \Phi}{\delta \theta}\right]_{R=R_{I}} .
$$

This is approximately true throughout the high-latitude region. Interestingly, the same equation is derived if the Hall 

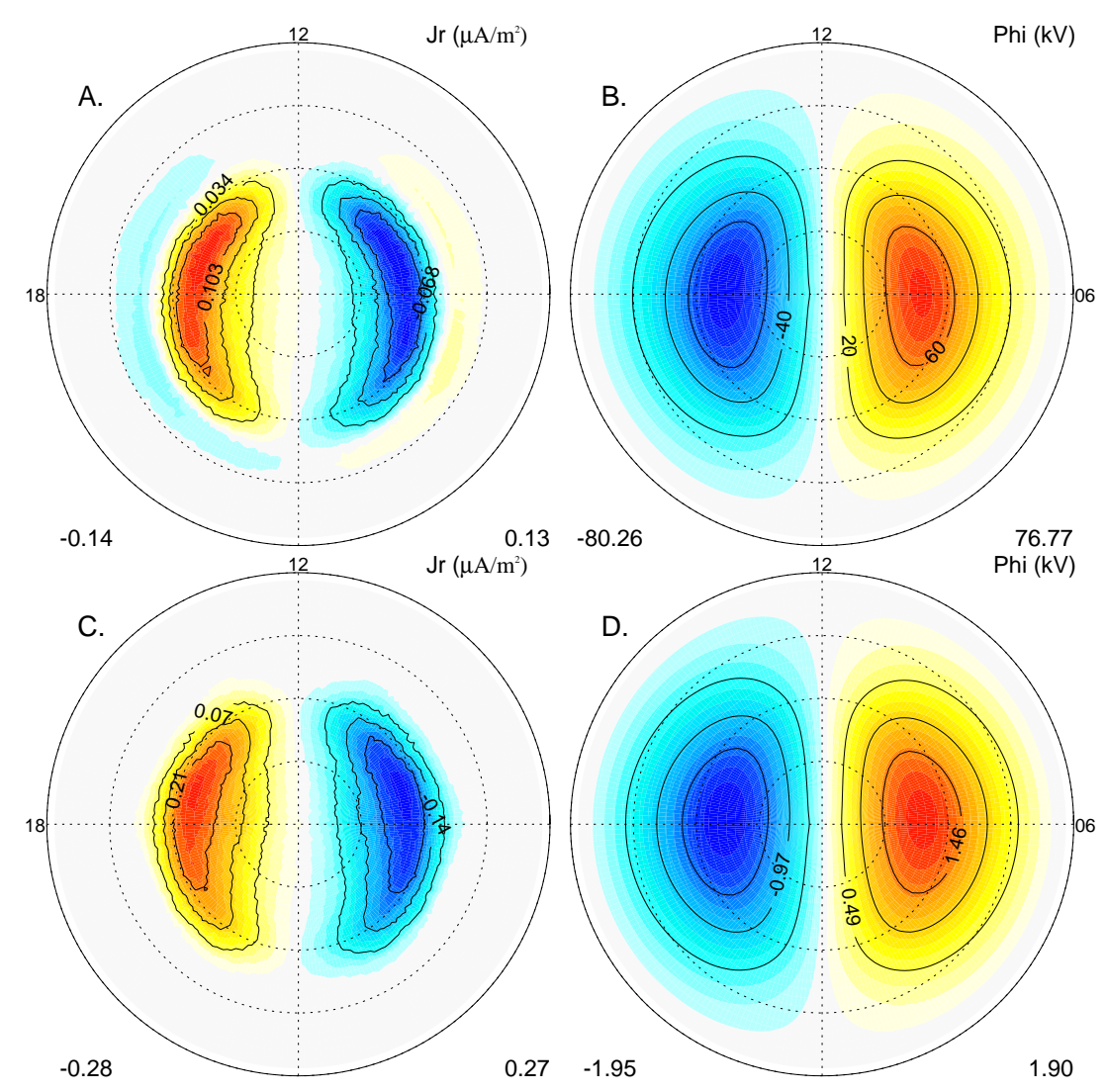

Plate 1. The $(\mathbf{A}, \mathbf{C})$ ionospheric field-aligned current and $(\mathbf{B}, \mathbf{D})$ potential patterns, plotted in magnetic latitude - magnetic local time coordinates, with noon being at the top and dawn to the right. The outer circle is $50^{\circ}$ latitude, while the other rings mark $10^{\circ}$ latitude. The MHD model was run with a Pedersen conductance of 1 mho (A, B) and 100 mhos (C, D). The color scale of each plot is independent of the other plots, while the maximum and minimum values are shown below each plot to the right and left, respectively.

conductance is nonzero and uniform and $\varepsilon=0$. We investigate the influence of the nonzero $\varepsilon$ and Hall conductance in the next section.

While the MHD code uses Eq. (1) to solve for the electric potential, Eq. (5) is easier to understand in this simplified regime. Plate 1A and B shows steady-state field-aligned current and electric potential patterns when the Pedersen conductance is set to $1 \mathrm{mho}$, while Plate $1 \mathrm{C}$ and $\mathrm{D}$ shows the solutions when Pedersen conductance is 100 mhos. The simulation was carried out with $B_{z}=-5.0 \mathrm{nT}, V_{x}=-500 \mathrm{~km} / \mathrm{s}$, and $n=5 \mathrm{~cm}^{3}$, while all other components of the velocity and magnetic field were zero. The lowest latitude mapping of the field-aligned currents is $\sim 58^{\circ}$ latitude. Each figure in Plate 1 show the patterns on different scales, so while the patterns are very similar, the magnitudes are quite different. The field-aligned currents show a strong region 1 current, but little region 2 current system. The 1 mho case does have small region 2 currents, but they are quite small compared to the region 1 currents. For the 100 mho case, there are essentially no region 2 currents. The reason for this becomes clear when the magnetospheric solutions are investigated. Plate 2 shows plots of the pressure and stream traces of the velocity in the equatorial plane for the 1 mho and 100 mho simulations. There are distinct differences between the simulations.
The first difference between the simulation results is in the flow pattern in the inner magnetosphere. The 100 mho simulation has much lower flow speeds coming in from the tail, so the corotation region is much further extended out into the magnetosphere. The 1 mho simulation shows the corotation to be much more confined to the near-Earth region, since the flow is much stronger coming in from the tail. This difference in flow speeds is the cause of the second difference: the pressure earthward of the divergent flow region in the tail (i.e. around $X=-7 R_{E}$ ) is stronger in the 1 mho simulation than in the 100 mho simulation. The strong flow breaking in the 1 mho simulation creates a large pressure buildup in the inner magnetosphere. Because the strength of the region 2 currents are strongly dependent upon the magnitude of the pressure in this region, the 1 mho simulation has a much higher region 2 current than the 100 mho simulation.

Similar runs were conducted using uniform Pedersen conductances of 2.5, 5.0, 10.0, 25.0, and 50.0 mhos. Figures 1a and $\mathrm{b}$ shows the maximum field-aligned current and cross polar cap potential versus the Pedersen conductance for each of these runs, respectively. These plots show that as the conductance is increased, the potential falls, while the fieldaligned current increases. This trend is easy to understand if we consider the simplified equation $J=\sigma_{p} E$. If we as- 

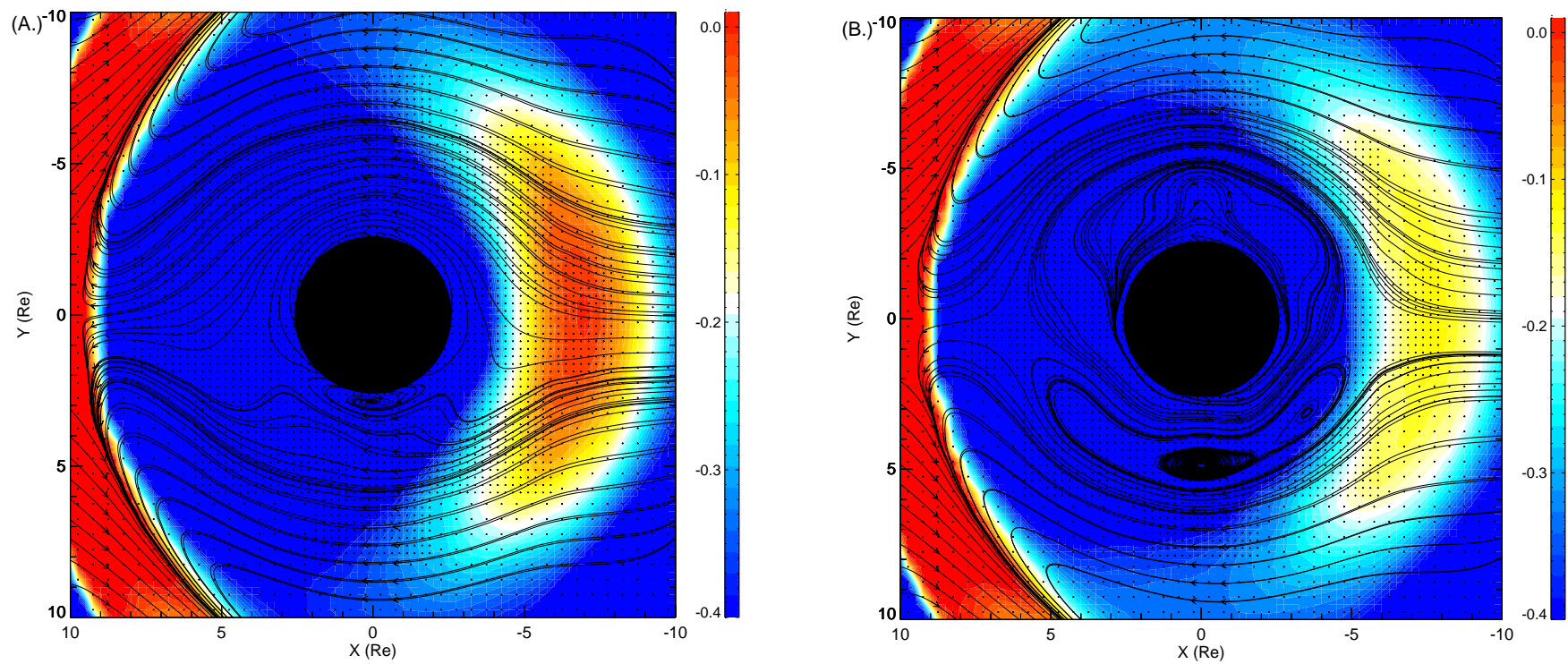

Plate 2. Color contours of the $\log$ of the pressure (in $\mathrm{nP}$ ) in the equatorial plane of the magnetosphere, with stream traces of the in-plane velocity overplotted. (A) shows the 1 mho simulation results while (B) shows the 100 mho simulation results.
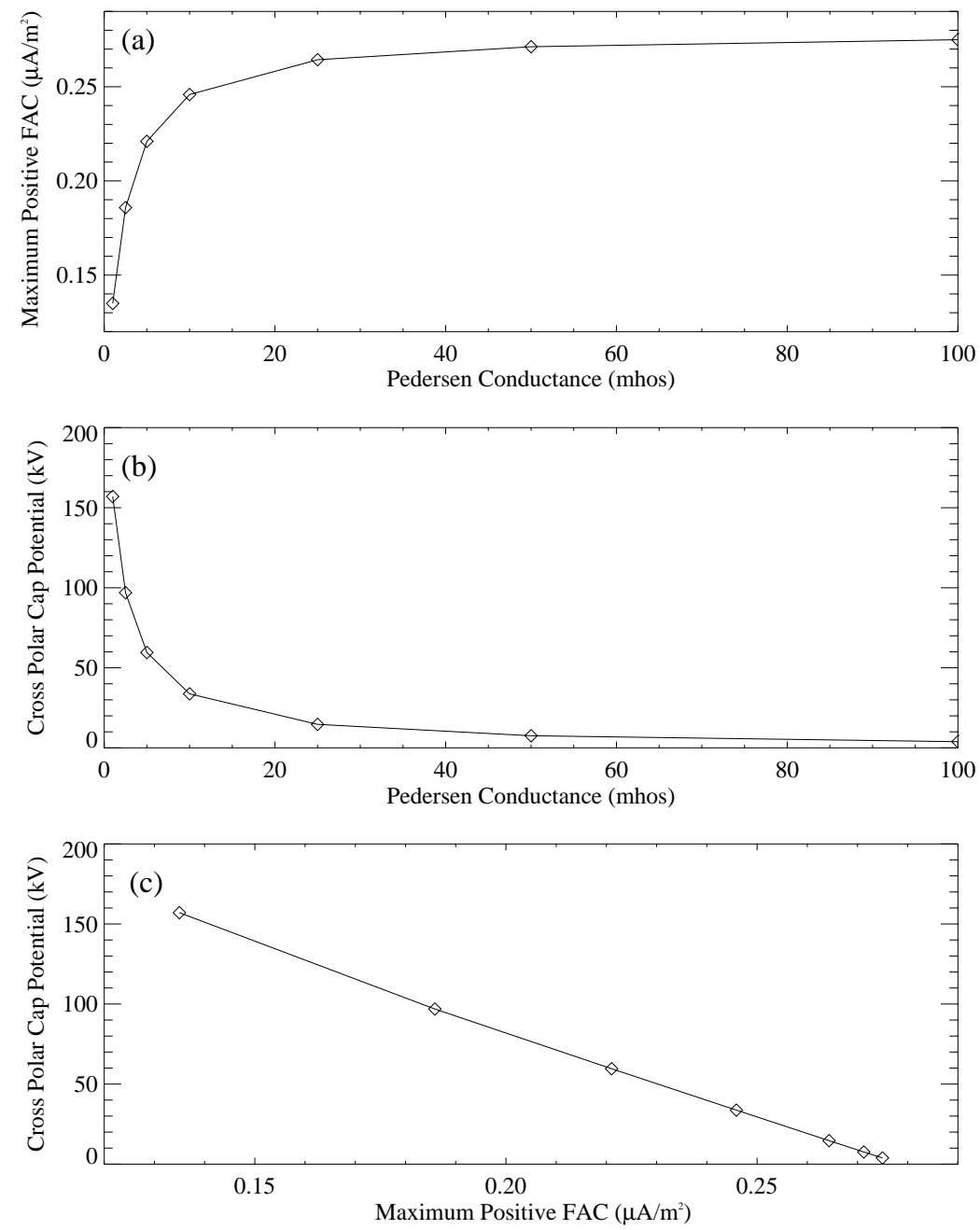

Fig. 1. (a) The maximum field-aligned current into the ionosphere and (b) the cross polar cap potential versus the Pedersen conductance. (c) The maximum field-aligned current into the ionosphere versus the cross polar cap potential. 


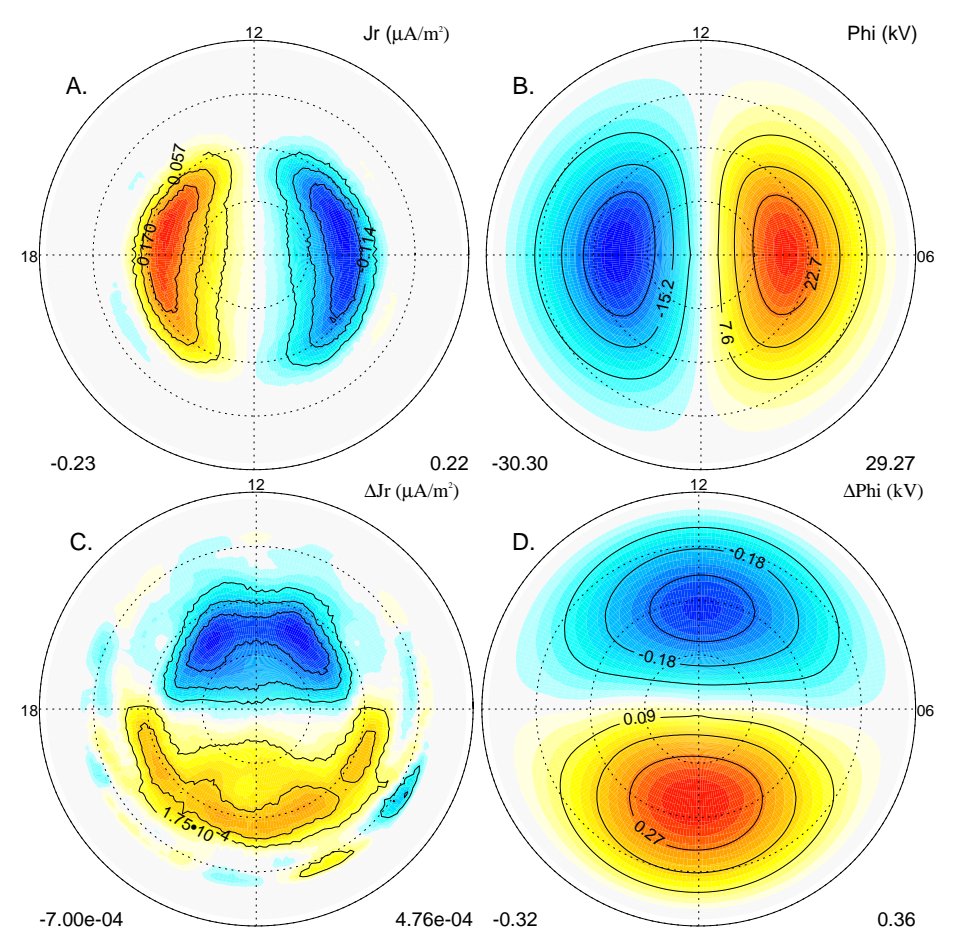

Plate 3. The (A) ionospheric field-aligned current and (B) potential patterns, for a simulation with the Pedersen conductance at 5 mho, and the Hall conductance of 0 mho. (C) The difference between (a) and the field-aligned current solution with a 10 mho uniform Hall conductance and (D) the same for the electic potential. Each plot is in the same coordinate system as Plate 1. Once again, each plot has an independent color scale, while the maximum and minimum values are indicated below the plots.

sume that the magnetosphere is a current generator, such that $J$ is constant, and we increase $\sigma_{p}, E$ must decrease linearly. On the other hand, if the magnetosphere acts as a voltage generator, such that $E$ is constant, when $\sigma_{p}$ is increased, $J$ must decrease. While the general trends are similar, it has been shown that the magnetosphere acts neither as a current nor as a voltage generator (Fedder and Lyon, 1987). We show a similar trend, as was shown by Fedder and Lyon (1987) in Fig. 1c: as $\sigma_{p}$ is increased, both $J$ and $E$ change together. These results are quiet similar to those of Fedder and Lyon (1987), although the strength of the cross polar cap potentials are significantly lower in this study (the reason for the difference is unknown and beyond the scope of the current study). The linear trend in the relationship between the potential and the field-aligned current is observed in both cases. Fedder and Lyon (1987) extrapolated their results with 5.0 mho, while we show that the extrapolation is valid up to at least 100 mho.

In the simulation run with 5 mhos Pedersen conductance, the cross polar cap potential is $59.6 \mathrm{kV}$, which is lower than the Weimer (1996) model, which shows a cross polar cap potential of $\sim 75 \mathrm{kV}$ for a $B_{z}=-5 \mathrm{nT}$ IMF orientation. The simulation with 2.5 mhos has a cross polar cap potential of $\sim 100 \mathrm{kV}$, so a constant Pedersen conductance of $\sim 4$ mhos would reproduce the (Weimer, 1996) cross polar cap potential for $B_{z}=-5 \mathrm{nT}$.

\subsection{Nonzero constant Hall conductance}

Equation (5) shows that if the magnetic field lines are radial, then a constant Hall conductance would have no influence over the ionospheric potential pattern. In reality, the magnetic field is much closer to a dipole:

$B_{R}=\left[2 B_{0} \cos \theta\right]_{R=R_{I}}$

$B_{\theta}=\left[B_{0} \sin \theta\right]_{R=R_{I}}$,

where $B_{0}$ is the magnetic field at the equator in the ionosphere. The sin and cos of the dipole tilt can then be expressed as

$$
\begin{aligned}
& \sin \varepsilon=\frac{\sin \theta}{\left(1+3 \cos ^{2} \theta\right)^{1 / 2}} \\
& \cos \varepsilon=-\frac{2 \cos \theta}{\left(1+3 \cos ^{2} \theta\right)^{1 / 2}}
\end{aligned}
$$

and assuming that the conductances are uniform, Eq. (3) becomes:

$$
\begin{aligned}
& j_{R}\left(R_{I}, \theta, \psi\right)= \\
& \frac{1}{R_{I}^{2}}\left[\frac{\Sigma_{0} \Sigma_{P}}{C} \frac{\delta^{2} \Phi}{\delta \theta^{2}}+\right. \\
& \left\{\frac{\Sigma_{P}}{\sin ^{2} \theta}+\frac{\Sigma_{H}^{2}}{C\left(1+3 \cos ^{2} \theta\right)}\right\} \frac{\delta^{2} \Phi}{\delta \psi^{2}}+
\end{aligned}
$$




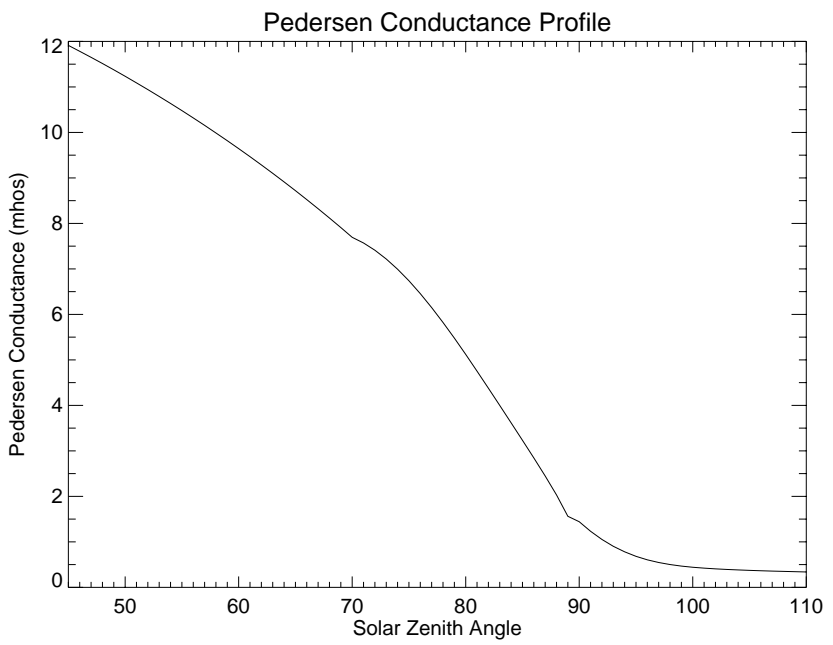

Fig. 2. A plot of the Pedersen conductance as a function of solar zenith angle. This shows the solar driven conductance, a scattering term which causes the conductance to be smoother across the terminator, and a nightside constant conductance. The squares of the conductances are added and the square root is taken to derive the total Pedersen conductance.

$$
\begin{aligned}
& \frac{\delta \Phi}{\delta \theta}\left\{\frac{\Sigma_{0} \Sigma_{P} \cos \varepsilon \sin \varepsilon\left(\Sigma_{P}-2 \Sigma_{0}\right)}{C^{2}\left(1+3 \cos ^{2} \theta\right)}+\frac{\Sigma_{0} \Sigma_{P} \cos \theta}{C \sin \theta}\right\}+ \\
& \left.\frac{2 \Sigma_{0} \Sigma_{H}}{C\left(1+3 \cos ^{2} \theta\right)^{3 / 2}} \frac{\delta \Phi}{\delta \psi}\left\{\frac{\left(\Sigma_{P}-2 \Sigma_{0}\right) \cos ^{2} \varepsilon}{c}-1\right\}\right]_{R=R_{I}} .
\end{aligned}
$$

Equation (10) shows that, when the magnetic field is considered to be a dipole, the electric potential does depend on the uniform ionospheric Hall conductance. This dependence is shown to be through the longitudinal gradient of the electric potential, which implies that the east-west electric fields are driving north-south Hall currents which close the magnetospheric field-aligned currents. Plate 3 shows this dependence, plotting the field-aligned current and electric potential pattern for the zero Hall case, and the difference between a simulation with 10 mhos Hall conductance and 0 mho Hall conductance. The difference is in the regions in which the potential has a gradient in the longitude (i.e. near noon and midnight), while there is no difference in the regions of strongest latitudinal gradient (i.e. dawn and dusk). The difference is only a fraction of a percent of the total strength, but as the pattern expands (e.g. during high magnetic activity), the difference is expected to grow, since the dipole tilt becomes more significant. In addition, when lowlatitude features are taken into account, such as the neutralwind dynamo, the Hall conductivity becomes important.

\section{Solar EUV conductance}

In the previous section, the conductance was estimated to be constant globally. While that may be approximately true locally, the conductance is known to have a strong day- night asymmetry. The solar illumination determines the dayside dynamics and structure of the thermosphere and ionosphere. Solar illumination in the wavelengths $\sim 10 \mathrm{~nm}-$ $100 \mathrm{~nm}$ produce $\mathrm{O}^{+}, \mathrm{O}_{2}^{+}, \mathrm{N}_{2}^{+}$, and $\mathrm{NO}^{+}$through various chemical reactions (e.g. Torr et al., 1979), many of which are directly driven by solar illumination. This means that they are strongly dependent upon the solar zenith angle of the given point and the composition of the atmosphere between the point and the Sun. In addition to the chemistry of the thermosphere-ionosphere system, it is important to take into account advection, diffusion, and other processes which are beyond the scope of any MHD model. Determining the true 3-D structure of the ionosphere globally is computationally time consuming, and, therefore, approximations are made.

For example, from the magnetospheric perspective, the ionosphere can be approximated to be a 2-D plane. The ionosphere can further be approximated to have a solar component and an auroral component. The solar component can be approximated as (Moen and Brekke, 1993):

$$
\begin{aligned}
& \left.\Sigma_{H}=F_{10.7}^{.53}(0.81 \cos (\zeta)+0.54 \sqrt{\cos (\zeta)})\right) \\
& \left.\Sigma_{P}=F_{10.7}^{.49}(0.34 \cos (\zeta)+0.93 \sqrt{\cos (\zeta)})\right),
\end{aligned}
$$

where $F_{10.7}$ is the solar flux intensity at $10.7 \mathrm{~cm}$, and $\zeta$ is the solar zenith angle.

Plate 4 shows simulation results in which there are only 3 sources of conductance: (1) solar EUV, (2) scattering of the sunlight across the terminator and (3) nightside "starlight" conductance. The nightside Pedersen conductance is estimated to be 0.25 mhos, which dominates on the nightside (neglecting the aurora, as is done here). The solar EUV strongly dominates on the dayside. All conductances within the code are summed together using a vector summation. For example, the total Hall conductance discussed below is:

$\Sigma_{\mathrm{H}}=\sqrt{\Sigma_{\mathrm{HEUV}}^{2}+\Sigma_{\mathrm{HScat}}^{2}+\Sigma_{\mathrm{HSL}}^{2}}$,

where $\Sigma_{\text {HEUV }}$ is the solar EUV Hall conductance (i.e. Moen and Brekke, 1993), $\Sigma_{\text {HScat }}$ is the scattered sunlight Hall conductance, and $\Sigma_{\mathrm{HSL}}$ is the starlight Hall conductance. Similar equations are used when considering the polar cap conductance and the auroral conductance.

Figure 2 shows a profile of the Pedersen conductance as a function of $\zeta$. This plot shows that the scattered sunlight causes the terminator to be much more diffuse than the sharp transition that one would expect with the Moen and Brekke (1993) formulation. It was found that with such a sharp transition, the slope of the potential would change dramatically across the terminator. With a more diffuse transition, the potential is much smoother across the terminator, which is more consistent with observations.

These runs were conducted without a Hall conductance to simplify the results. Two simulations were run: solar maximum (top) and solar minimum (bottom). The difference between these simulations are discussed below. In comparing the field-aligned currents and the potentials between simulations in which the conductance is constant (i.e. Plate 1) and 

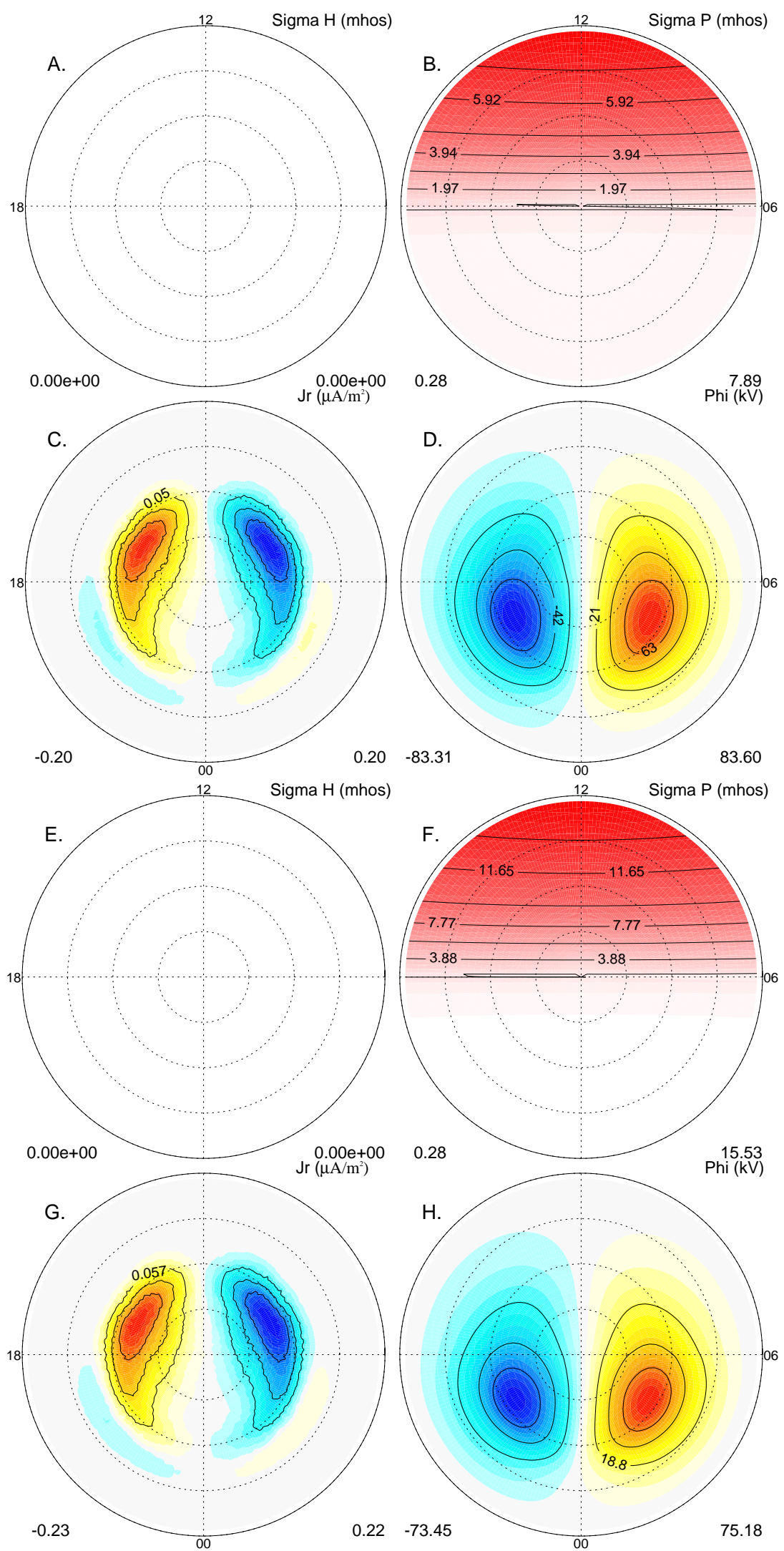

Plate 4. The top cluster of 4 plots (A-D) are the ionospheric results from a simulation during solar minimum, while the bottom 4 plots $(\mathbf{E}-\mathbf{H})$ are from solar maximum. The runs were conducted with the Hall conductance equal to 0. The plots are (A, E) the Hall conductance; (B, F) Pedersen conductance; $(\mathrm{C}, \mathrm{G})$ field-aligned currents; and $(\mathrm{D}, \mathrm{H})$ potential pattern. Each plot is in the same coordinate system as Plate 1 . 

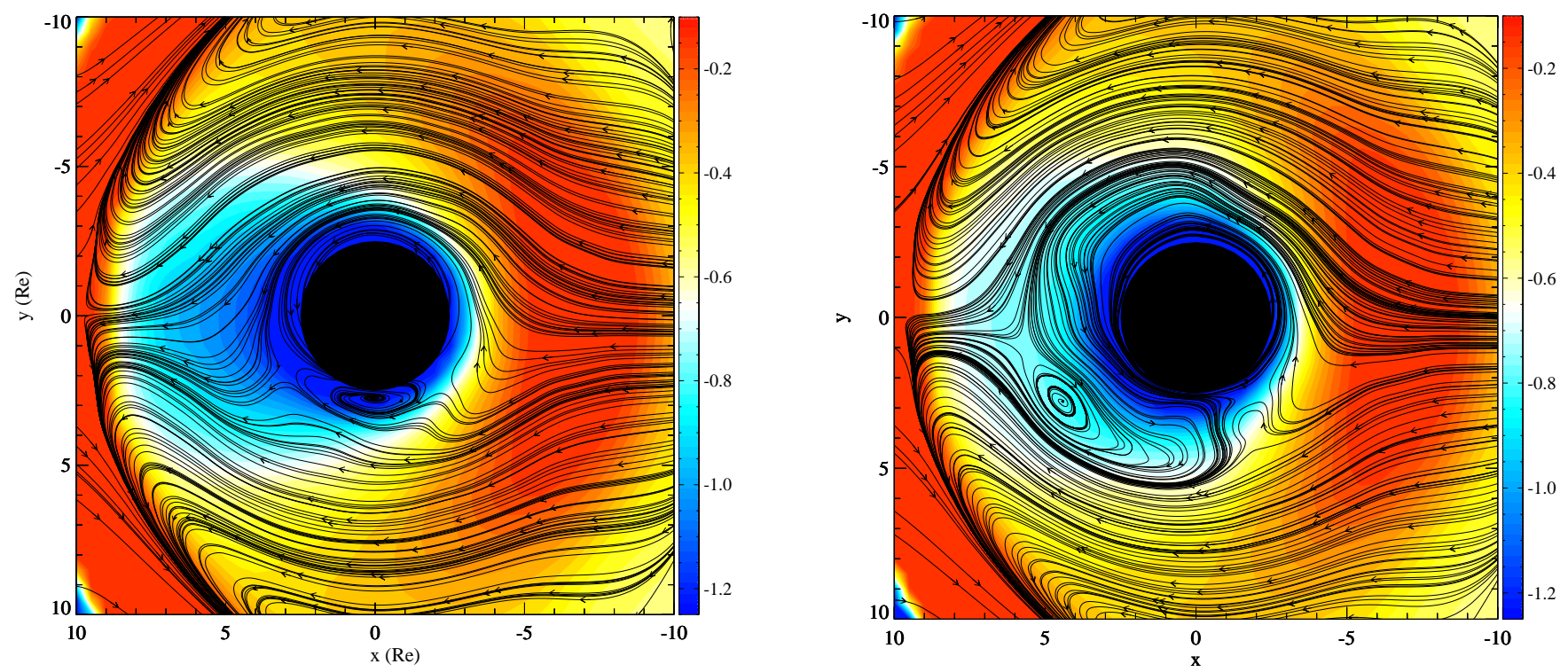

Plate 5. Flowlines in the magnetosphere in the $X-Y$ plane, neglecting $U_{z}$, over color contours of $\log (p)$, where the pressure is in nP. The top plot shows a simulation in which the Pedersen conductance was uniform at 1 mho (i.e. Plate 1A and B, Plate 1C and D), while the bottom plot shows a solar maximum simulation in which only the solar EUV was considered, corresponding to Plate 4E-H. The density of the streamlines does not correspond to the strength of the field.

those in which the solar EUV is dominant (i.e. Plate 4), there are clear differences. In the solar EUV case, the field-aligned current flows primarily on the dayside, while the potential is confined to the nightside. The reason for this is illustrated in Fig. 1: when the conductance is high (i.e. the dayside), the field-aligned current is large and the potential is small, and conversely, when the conductance is low (i.e. the nightside), the field-aligned current is low and the potential is large.

Plate 5 illustrates the magnetospheric difference between a constant conductance case and a solar EUV case. This plate shows streamline traces over the log of the pressure in the inner magnetosphere for a simulation with 1 mho constant conductance and a simulation with solar EUV dominating and a 0.25 mho constant nightside conductance. On the nightside, the pressures are quite similar, as are the flow patterns. This is expected, since on the nightside, the conductances are similar to each other. On the dayside, there are significant differences in the pressure and the flow pattern. The flow patterns shows that the corotation is dominant to a much larger radius in the solar EUV case than in the constant low conductance case. This is because the ionospheric flow velocities on the dayside are much smaller in the EUV case. This domination of corotation on the dayside in the solar EUV case causes a stagnation of the plasma flow further away from the inner boundary, which, in turn, causes a thermal pressure enhancement on the dayside.

\section{1 $\quad F_{10.7}$ dependence}

Plate 4 illustrates the difference between solar minimum and solar maximum. The top plots show simulation results with only solar EUV derived Pedersen conductances for an $F_{10.7}$ of 75.0 (solar minimum conditions), while the bottom group of plots shows a simulation in which the $F_{10.7}$ was 300 (solar maximum conditions). There is a factor of 2 difference in the dayside conductance from solar min to solar max. The field-aligned currents increase by $\sim 11 \%$, while the cross polar cap potential decreases by the same factor. This is not a significant change, considering that these simulations show the maximum effect in which the solar EUV over a solarcycle would contribute to the field-aligned current and potential differences. Any addition of auroral sources of conductance would serve to decrease this difference, such that there may be even less than a $10 \%$ difference between the potentials at solar minimum and maximum.

\subsection{Nightside conductance}

On the nightside of the Earth, there is very little to cause ionization, except for the particle precipitation at high latitudes. Star light and galactic sources can cause some F-region ionization, and is included in the ionospheric model here. This ionization is added as a uniform conductance over the entire globe. As this background conductance is raised to comparable levels as the solar illumination, the ionosphere becomes closer to a constant conductance model, as described in the previous section. When it is reduced towards zero, the currents close more and more on the dayside, and a strong electric field results on the nightside, as is illustrated in Plate 4. In the magnetosphere, one would expect that as the nightside conductance is increased, the flow velocity would decrease and the pressure would decrease, as was discussed in the constant conductance section above, and illustrated in Plate 2. 

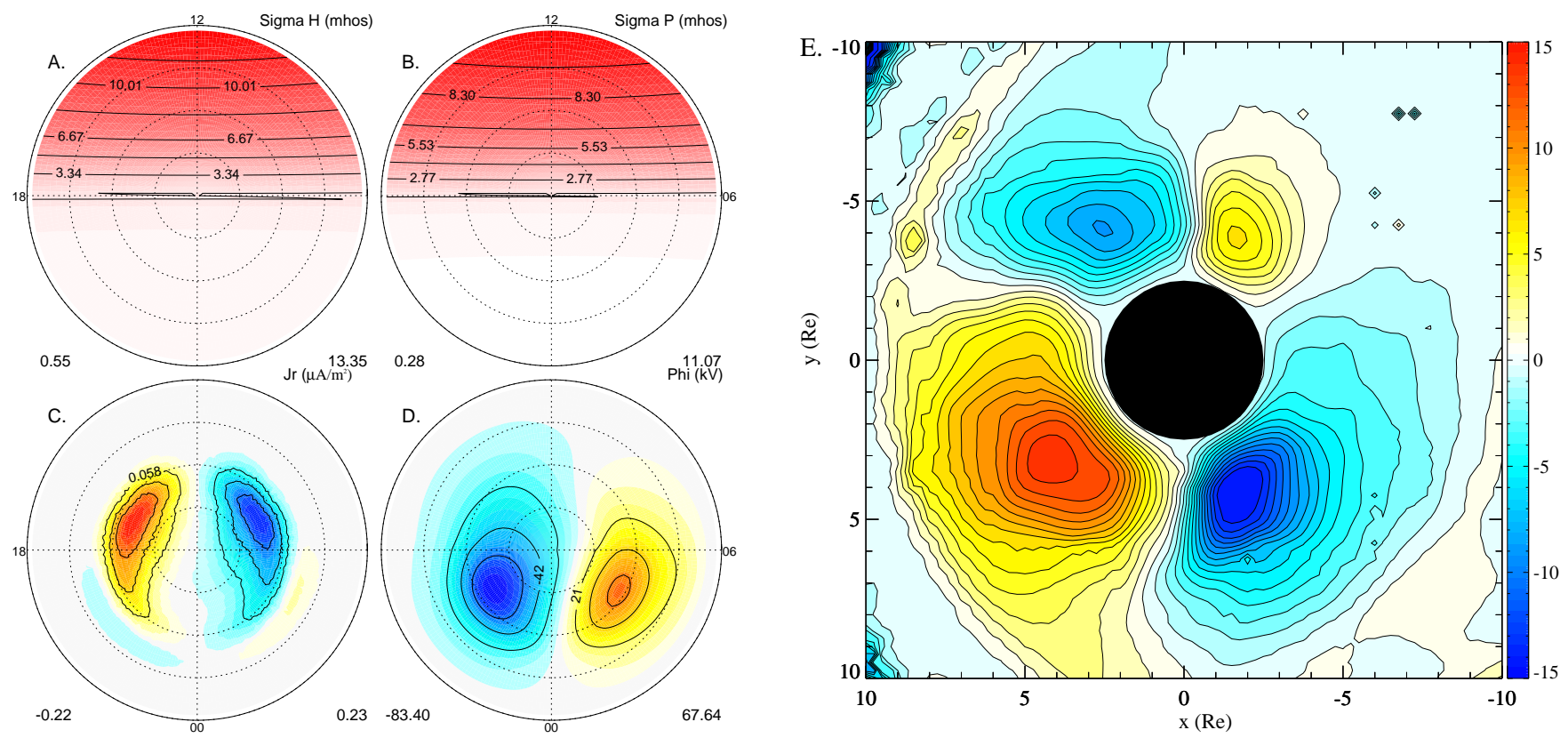

Plate 6. An ionospheric projection of the (A) Hall conductance, (B) Pedersen conductance, (C) FAC, and (D) potential, as shown in Plate 1, as well as $(\mathbf{E})$ a plot of the percentage difference in pressure in the inner magnetosphere between a simulation with and without solar derived Hall conductance in the ionosphere.

\subsection{Hall conductance effects}

The previous sections showed that a constant Hall conductance pattern does not effect the potential at high latitudes very stongly. This is not true when there is a strong gradient in the Hall conductance. Plate 6 shows the effect of the Hall conductance on the potential pattern: the symmetry across the noon-midnight meridian is broken, with the morning cell decreasing in size significantly. The solar EUV generated Hall conductance causes the potential pattern to rotate such that the throat is closer to 11:00 MLT instead of 12:00 MLT, while the negative cell in the midnight sector encroaches into the early morning sector.

In the magnetosphere, the convection pattern becomes more asymmetric, which, in turn, changes the pressure distribution. Plate 6 shows the percentage difference of the magnetospheric pressure distribution between runs with and without the Hall conductance. The differences are $\pm 15 \%$. The locations of the largest differences on the dusk side are approximately where stagnation regions exist, indicating that the stagnation regions have moved. On the dawn side, the differences are due the changes in the convection velocity sweeping away the plasma faster (slower), resulting in regions of decreased (increased) plasma pressure.

\subsection{Seasonal effects}

Because the main conductance on the dayside is driven by the solar zenith angle, seasonal effects are of primary importance when considering the conductance and the resulting electric potential pattern. This is because the Earth's magnetic dipole axis tilts $\sim 34^{\circ}$ towards and away from the Sun in the Northern Hemisphere during solstice conditions at certain times of the day. This tilt can cause the conductance to be quite high (or low) compared to the opposite magnetic hemisphere.

Plate 7 shows a cut of a magnetospheric simulation in the noon-midnight meridian. The Northern Hemisphere is tilting $34^{\circ}$ toward the Sun, while the Southern Hemisphere is tilting away from the Sun an equal angle. The northern and southern ionospheric field-aligned current, Hall and Pedersen conductance, and potential patterns are shown in Plate 8. All four of the patterns show a clear asymmetry between the two hemispheres. Because the conductance pattern is so different between the hemispheres (and is held constant in time), the currents adjust to flow more through the Northern Hemisphere. This readjustment is not enough to allow for the potentials to equalize, so there exists a significant difference in the cross polar cap potentials in the two hemispheres.

Plate 7 illustrates why this may be the case in this simulation - the distance which has to be travelled from the merging region on the dayside to the reconnection region on the nightside in the Northern Hemisphere is shorter than the distance which has to be travelled in the Southern Hemisphere. This difference in the distance implies that the Northern Hemisphere field lines would need to move slower, in order to travel to the reconnection site in the same time as the field lines in the Southern Hemisphere.

A hemispheric difference this large is not commonly observed. Papitashvili and Rich (2002) indicate that a potential difference does exist between the summer and winter hemispheres, but is much smaller than that observed here. Plate 9 shows ionospheric potential patterns derived from the 
Weimer (1996) electric potential model for the same time period as shown in Plate 8. This model was derived from Dynamics Explorer data of ion flows, and would therefore show a large asymmetry, if one existed. The Southern Hemisphere does show a $17 \%$ larger cross polar cap potential than the Northern Hemisphere, while the MHD code shows a difference of $435 \%$. Also, the Weimer (1996) cross polar cap potential is approximately halfway between the northern and southern MHD results, leading to the conclusion that the true value should be between the two.

The MHD code shows that the conductance in the Southern Hemisphere is approximately uniform (but low) in the polar cap, while in the northern polar cap there is a day to night gradient. This causes the potential in the Northern Hemisphere to be pushed towards the nightside, while the potential maximum and minimum in the Southern Hemisphere are near the dawn-dusk meridian. In the Weimer (1996) patterns, the trend is different - the Northern Hemisphere potentials are on the dayside, while the Southern Hemisphere potentials on the nightside. The location of the potentials on the nightside in the Southern Hemisphere indicate a day to night gradient in the conductance, while the location of the potentials on the dayside in the Northern Hemisphere imply that there may in fact be more conductance on the nightside than on the dayside, which implies an auroral oval. This will be discussed in the next section.

The lack of a strong north-south asymmetry in the Weimer (1996) patterns implies that these specific MHD simulations are missing some important physics:

- The ionospheric conductance dependence on solar EUV may be wrong for large solar zenith angles. We may be underestimating the conductance behind the terminator by a large amount. If this is true, the conductance may be more equalized, thereby reducing the potential in the winter hemisphere. More studies of the conductance at very high latitudes would have to be examined to determine if this is true.

- Adding an auroral oval will help the winter-summer asymmetry. In addition, Liou et al. (1997) showed that the auroral brightness is dependent upon season, with the winter months being brighter than the summer months. This seasonal effect would serve to equalize the potentials. Ridley et al. (2002) discuss this seasonal effect on the conductance in more detail. They find that by increasing the total energy flux in the auroral oval and polar cap in the winter hemisphere, the potential becomes much more symmetric.

- If the potentials in the two hemispheres are approximately equal, as indicated in Plate 9, then the magnetic field structure in the tail shown in Plate 7 may be wrong. This plate implies that during the Northern Hemisphere summer, the current sheet may be lifted out of the equatorial plane by as much as $5 R_{E}$.

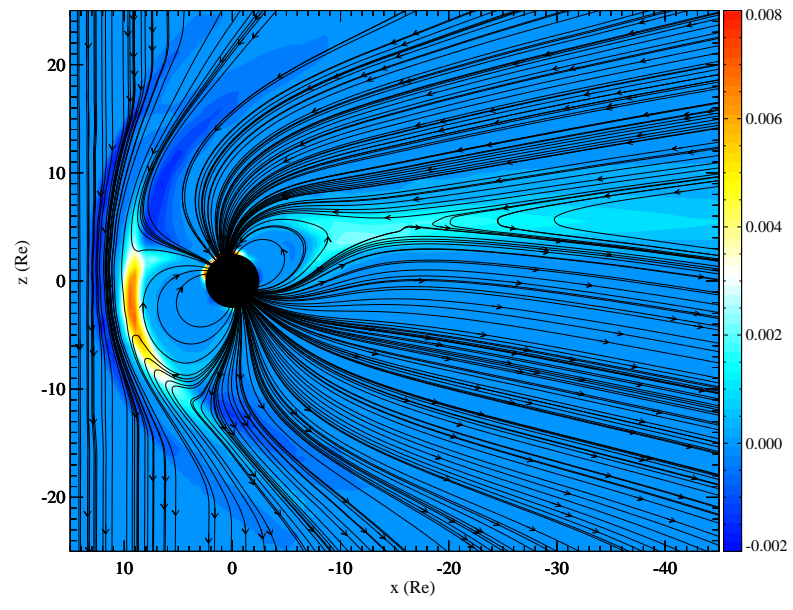

Plate 7. Traces of the magnetic field in the $X-Z$ plane, ignoring $B_{y}$, over $J_{y}$ in units of $\mu A / m^{2}$. This figure illustrates a summer solstice condition in at 16:45 UT, in which the Northern Hemisphere magnetic pole is pointed further towards the Sun. The density of the fields lines does not correspond to the strength of the field.

\section{High latitude conductance}

While solar EUV is quite important in determining the structure on the conductance, field-aligned current, and potential on the dayside, a number of processes are still excluded when the solar source is the only one considered. Namely, the highlatitude particle precipitation is quite important. In this section, auroral precipitation is investigated, as well as the polar rain, which adds a small amount of conductance within the polar cap.

\subsection{Auroral conductance}

The aurora is made up of precipitating electrons, and ions to a lesser extent, which cause ionization in the thermosphere, mesosphere, and sometimes the stratosphere (e.g. Frahm et al., 1997; Galand et al., 2001). The electrons and ions then collide with the neutrals forming Hall and Pedersen conductivities. Many models of precipitating particles exist (e.g. Fuller-Rowell and Evans, 1987; Ridley et al., 1999). In addition, a number of studies have analytically determined the relationship between precipitating particles and local height integrated conductances (e.g. Robinson et al., 1987; Lummerzheim et al., 1991).

In MHD codes, there are no energetic particles, so no true auroral precipitation can be specified. Different modelers use different approaches to determine the auroral precipitation. For example, Raeder et al. (1998) describes a model which has both a diffuse aurora, which is specified by the plasma temperature in the plasma sheet, and a discrete aurora, which is specified by a relationship between upward field-aligned current and the energy of the downward precipitating electrons (Knight, 1972).

The Knight (1972) relationship works specifically for upward field-aligned current regions. This means that it is 
A. J. Ridley et al.: Ionospheric conductance influence on magnetospheric configuration
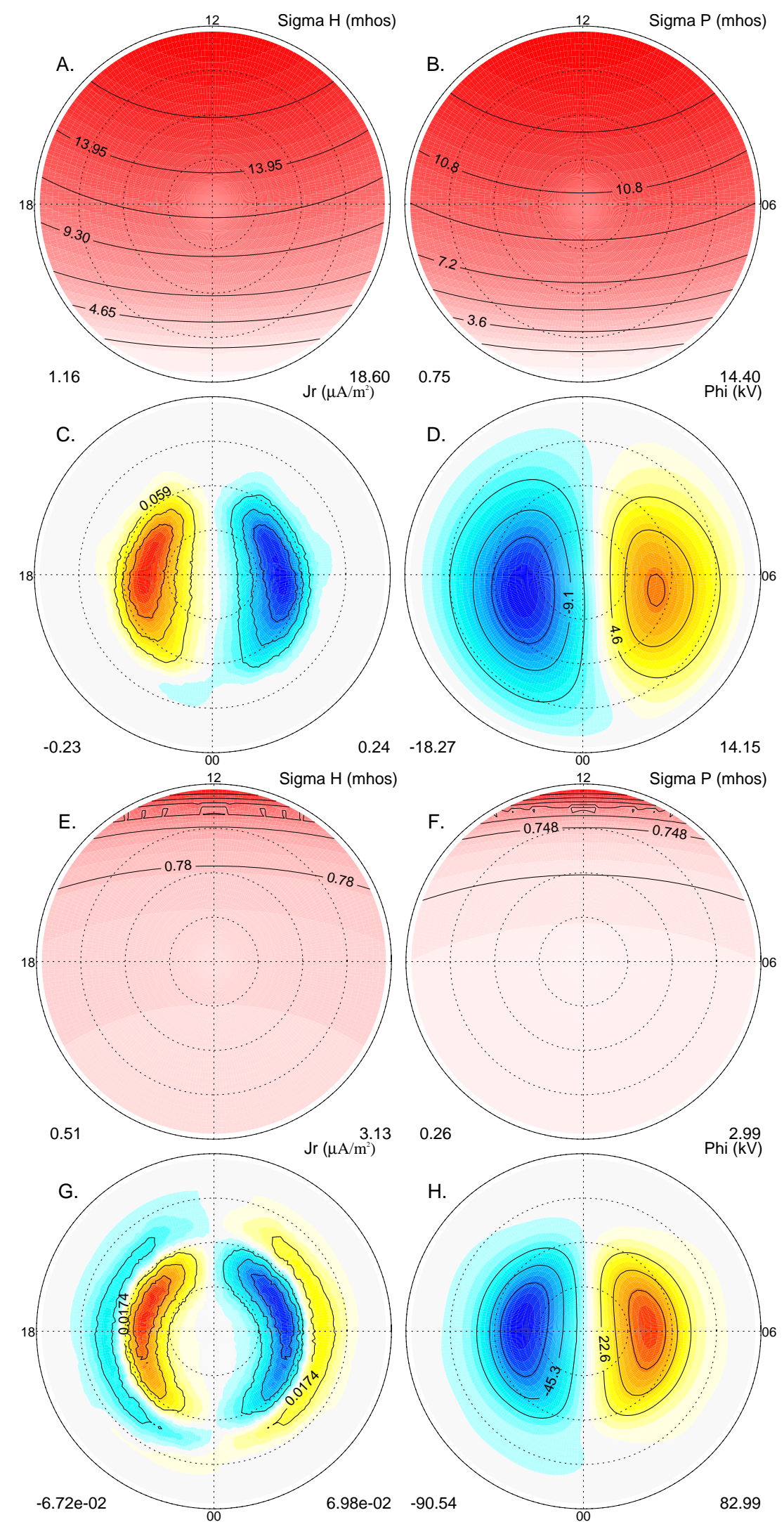

Plate 8. Ionospheric results for summer (A-D) and winter conditions (E-H), corresponding to Plate 7. This figure is in the same format as Plate 4. 

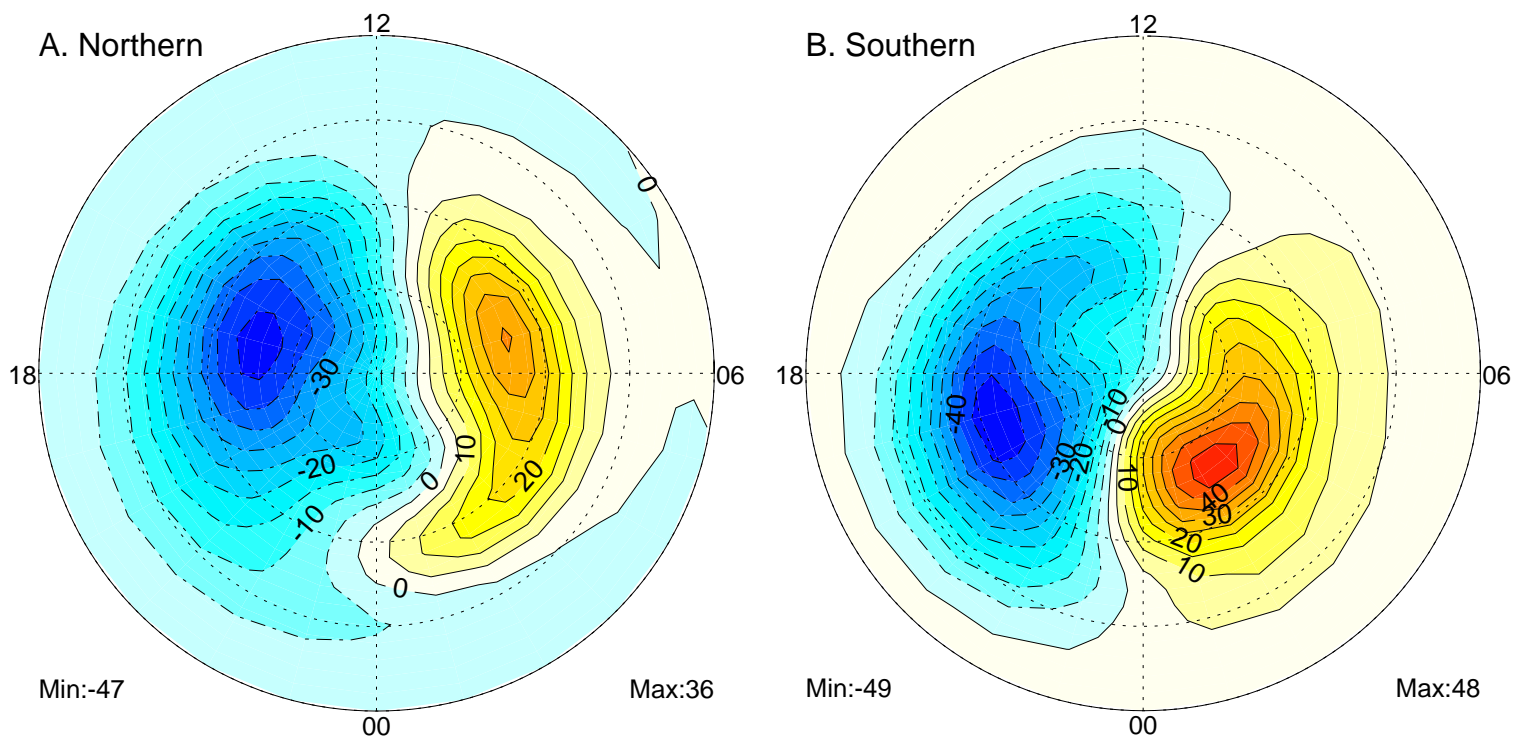

Plate 9. Ionospheric potential patterns from the Weimer (1996) model for (A) summer and (B) winter hemispheres during the same period as in Plate 8.
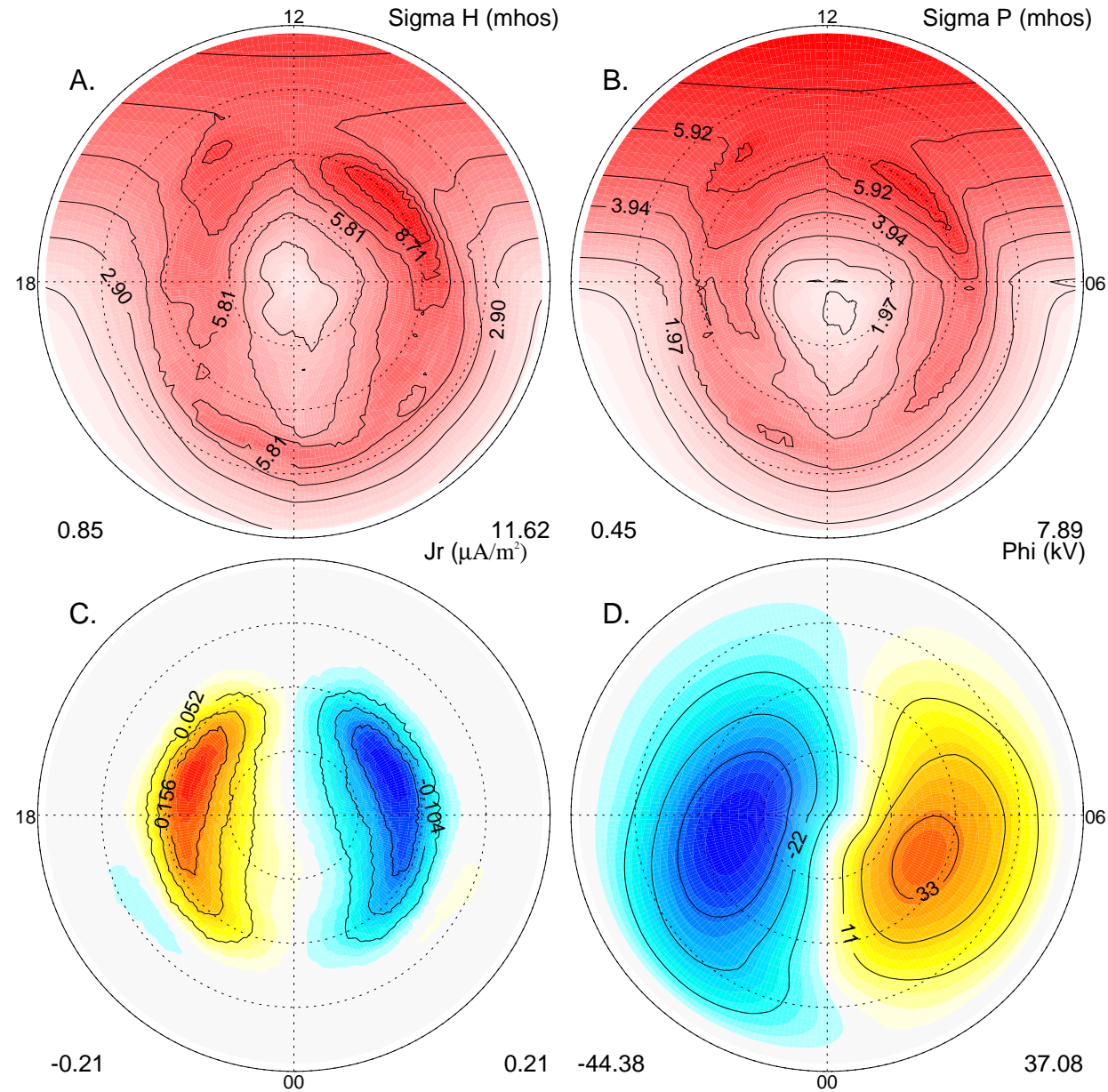

Plate 10. Ionospheric results for equinox conditions with a field-aligned current dependent auroral oval. This figure is in a similar format as Plate 4. 


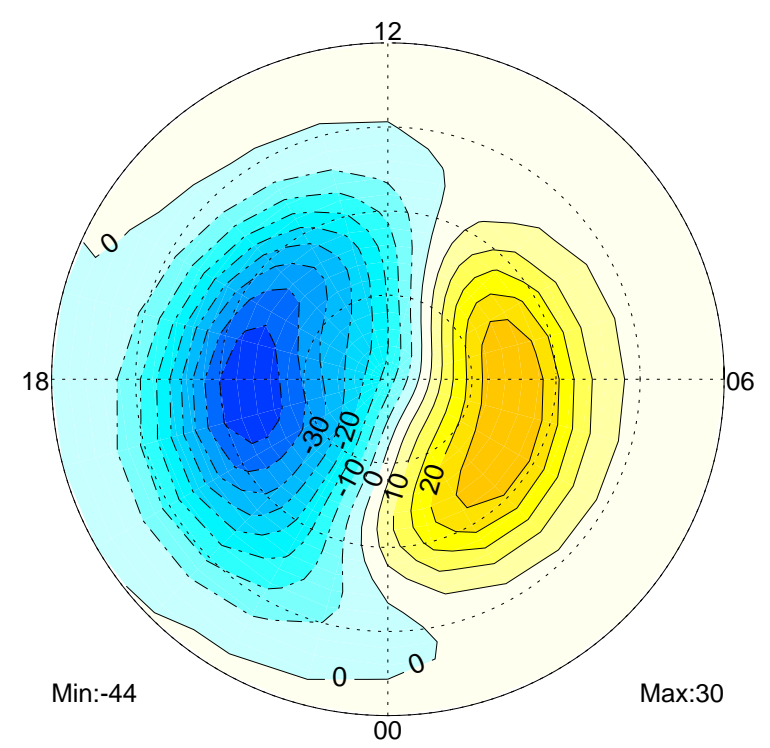

Plate 11. Ionospheric potential patterns from the Weimer (1996) model for equinox conditions during the same period as in Plate 10.

applicable only to region 1 currents on the dusk side and region 2 currents on the dawn side. In order to obtain a complete oval, the region 2 currents must be modeled correctly. Because this is not done accurately within most MHD models, the Knight (1972) relationship cannot be accurately applied in the dawn sector. In some models, the diffuse aurora may cover this missing physics. In other models, comparisons between imagery and the currents were used to determine where the conductance should be placed.

In the MHD model described here, a different approach is used. While this approach is not based on a first-principle description of the acceleration region or the diffuse aurora, it provides methodology for accurately calculating the auroral precipitation from the field-aligned currents. An emprical relationship was derived between the field-aligned currents and the local Hall and Pedersen conductance. This relationship was derived using the assimilative mapping of ionospheric electrodynamics (AMIE) technique (Richmond and Kamide, 1988). The AMIE technique was run at a one-minute cadence for the entire month of January 1997, using 154 magnetometers. This resulted in almost 45000 2-dimensional maps of Hall and Pedersen conductance, as well as fieldaligned currents. The conductance derived from AMIE was mainly driven by the Ahn et al. (1998) formulation, which relates ground-based magnetic perturbations to the Hall and Pedersen conductances. For each grid point, a relationship in the form of:

$\Sigma=\Sigma_{0} e^{-A J_{\|}}$,

was determined, where $\Sigma$ represents either the Hall or Pedersen conductance, and $\Sigma_{0}$ and $A$ are independent of the magnitude of $J_{\|}$, but depend on whether the current is up or down and the location. The gridded values of $\Sigma_{0}$ and $A$ are then used in the MHD code to relate the field-aligned current $\left(J_{\|}\right)$ to the Hall and Pedersen conductances.

This relationship is illustrated in Plate 10. These conductances have some features which are expected and some which are not expected. For example, the local maximum in the auroral oval at 09:00 MLT is reproduced in statistical patterns such as that shown in Hardy et al. (1987). A significant difference is the lack of conductance near midnight. This is because there are limited currents flowing in the midnight sector in the MHD simulation (and zero currents flowing right at midnight). Because the relationship given in Eq. (14) relates the conductance directly to the current, when there is no current, there is little precipitation-induced conductance. During time-dependent runs, when substorms are occurring, the current is expected to increase, which will then cause the conductance to increase.

As a comparison, the Weimer (1996) Northern Hemisphere pattern is shown in Plate 11. The Weimer (1996) pattern was derived for the same day and time of day as the MHD results shown in Plate 10. A remarkable agreement is observed between the two models.

Because the peak of the auroral oval is not near midnight, as predicted by empirical models, the auroral oval does not effect the magnetosphere as much as one may expect. The flow patterns and the pressure distribution in the $Z=0$ plane look remarkably like those shown in Plate 5B, with only small differences. Studies of the effects of the auroral oval on time-dependent simulation will follow.

It was suggested earlier in the study that an auroral oval would help to equalize the ionospheric potential in the summer and winter hemispheres during periods of strong dipole tilt $\left(\right.$ i.e. $35^{\circ}$ ), such as that shown in Plates 7 and 8 . There is a problem with this, given the formulation for the currentdependent aurora described above: the current flowing into the summer hemisphere is larger than that current flowing into the winter hemisphere. This will cause the auroral conductance to be larger in the summer hemisphere than in the winter hemisphere, which will not help to equalize the electric potentials. The validation study by Ridley et al. (2002) showed that there needs to be a seasonal dependence upon the strength of the auroral oval, to better equalize the potential between the hemispheres.

Plate 13 shows the ionospheric results with a seasonal dependence upon the strength of the aurora, a smearing of the oval, to put more conductance in the midnight region, and a uniform conductance of 1 (2) mho(s) Pedersen (Hall) added to the EUV generated conductance, as shown in Plate 8 (the background conductance was added to make the solution more stable, but does not contribute to the differences between the hemispheres). These figures show that the cross polar cap potential in the summer hemisphere is reduced from $32 \mathrm{kV}$ to $22 \mathrm{kV}$, while the winter potential is reduced from $174 \mathrm{kV}$ to $64 \mathrm{kV}$. This makes the hemispheric potentials much closer together, as is expected. 

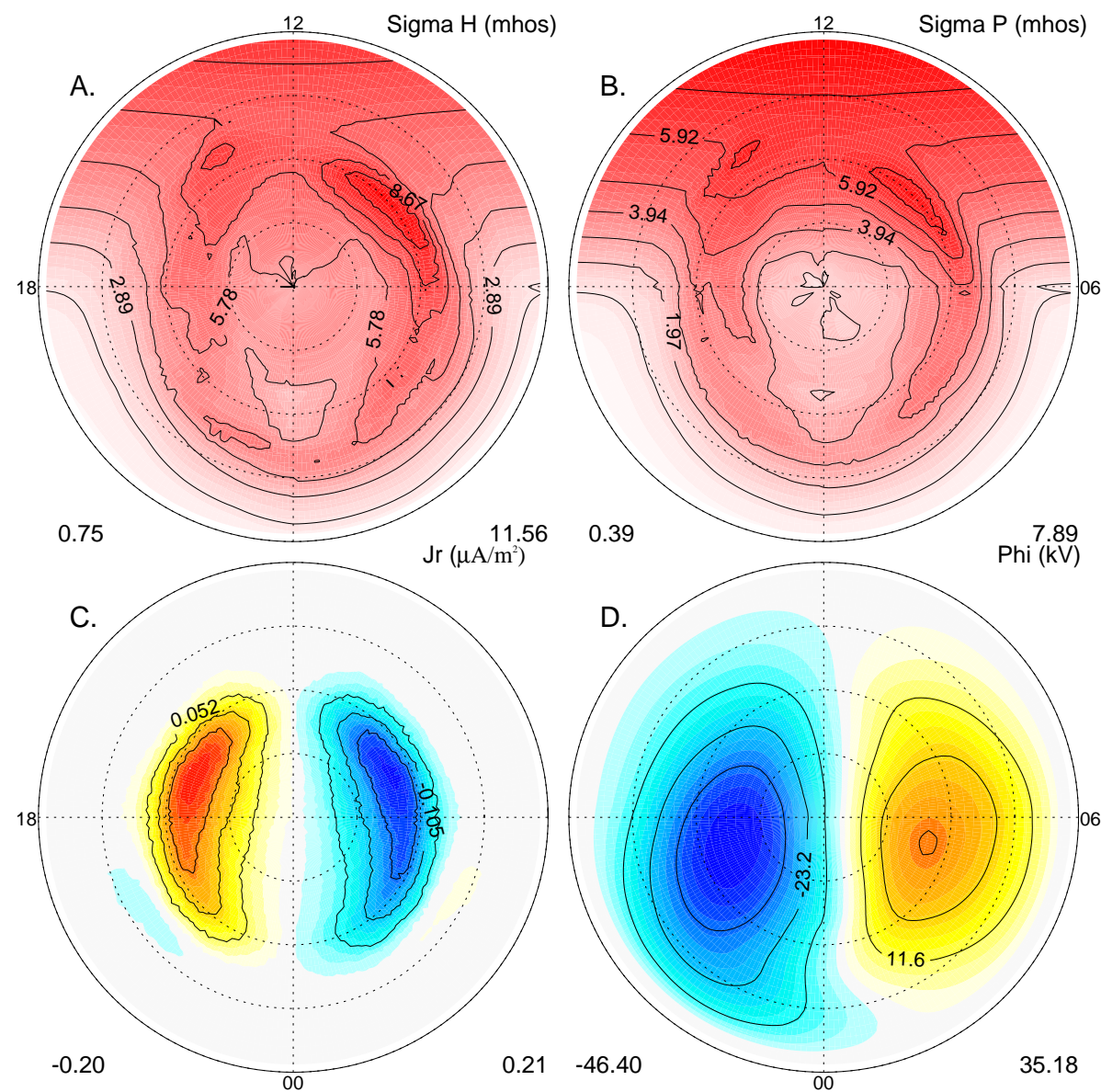

Plate 12. Ionospheric results for equinox conditions with a field-aligned current dependent auroral oval, and a constant polar cap conductance of 2.5 mhos added. This figure is in a similar format as Plate 4.

\subsection{Polar cap conductance}

Within the polar cap, there exists polar rain, which is an approximate uniform drizzling of electrons (Newell and Meng, 1992). This low energy electron precipitation can cause a small F-region conductance to form. Typically, the Pedersen conductance in the polar cap is on the order of $1 \mathrm{mho}$, but the authors are unaware of any published studies which show this value.

Plate 12 shows a simulation with the same parameters as the run shown in Plate 10, except that a constant polar cap conductance of 2.5 (5.0) mhos of Pedersen (Hall) conductance is added. The polar cap is defined as anything poleward of the peak particle precipitation flux in the auroral oval. In comparing Plates 12D and 10D, the potentials are quite similar. Including the polar cap conductance tends to move the throat back towards noon, and tends to remove some of the dawn-dusk asymmetry caused by the day-to-night gradient in the Hall conductance. This is expected, since by adding a constant conductance, the influence of the gradient is reduced.

\section{Summary}

The magnetosphere is influenced by the ionosphere in a number of fundemental ways. This study focused on quantifying the influence of the ionospheric conductance on the magnetospheric configuration during steady IMF southward conditions. The results are summarized as follows:

- The steady-state magnetosphere acts neither as a current nor as a voltage generator, as described by Fedder and Lyon (1987). This study shows that it is true for both larger and smaller values of conductance than those specified in the study by Fedder and Lyon (1987).

- When it is assumed that the ionospheric conductance is uniform, the Hall conductance does not influence the potential pattern at high latitudes. Because the potential is dependent on the dip angle when applying a constant Hall conductance, the influence becomes larger at lower latitudes, but is still only a few percent. Considering the other uncertainties and limitations of the model, this is a negligible effect.

- The day-to-night gradient in the solar EUV generated conductance tends to push the potential onto the 

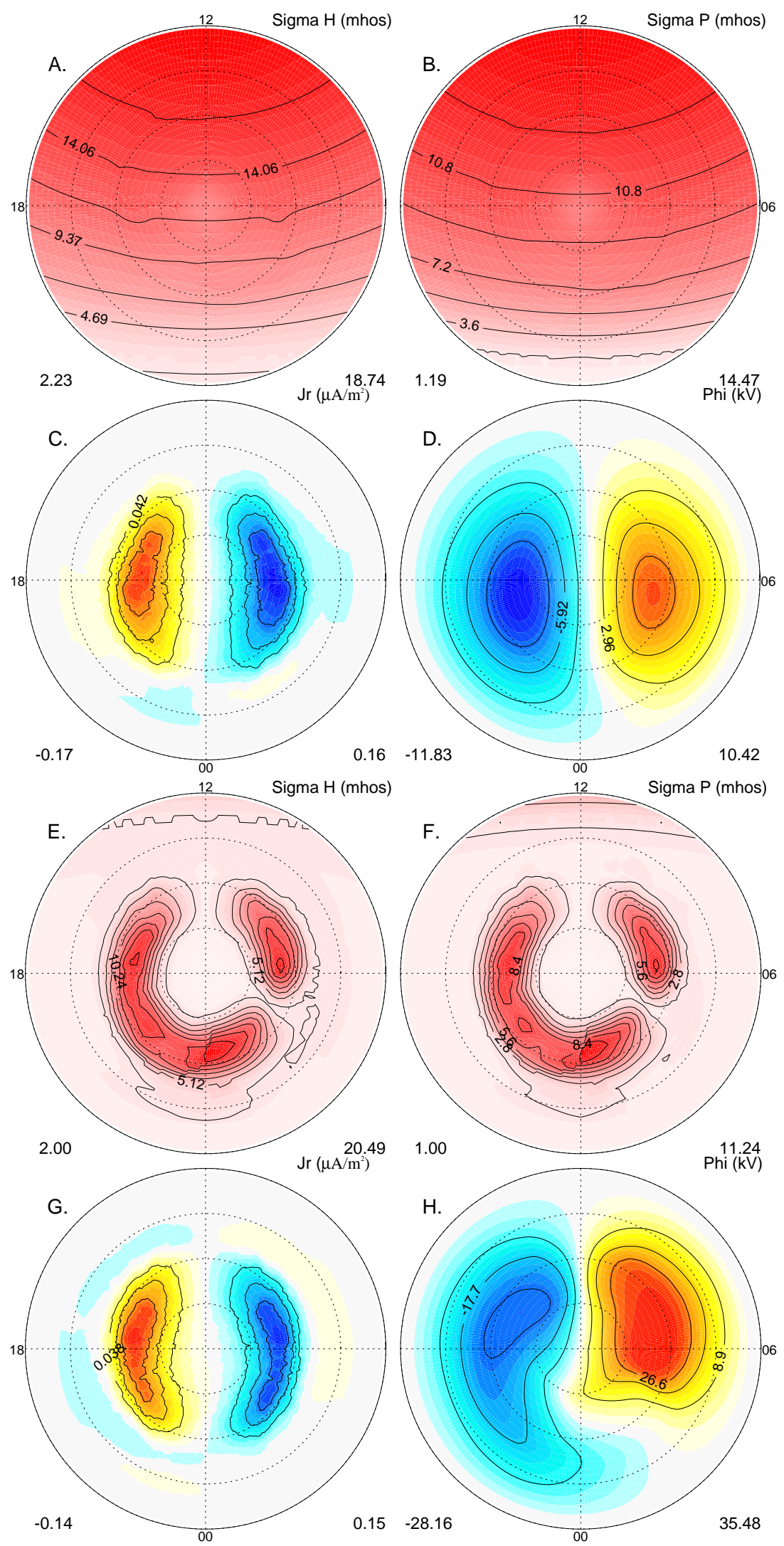

Plate 13. Ionospheric results for summer (A-D) and winter conditions (E-H), for a similar time as Plate 8, but with an auroral oval, a background conductance of 1 mho Pedersen, and a seasonal dependent polar cap conductance added. This figure is in the same format as Plate 4. 
nightside, while the field-aligned current flows on the dayside.

- If there is a strong asymmetry between the northern and Southern Hemisphere conductances, as exists in the MHD code during solstice conditions with only solar generated conductances, the tail is displaced above (below) the equatoral plane during the Northern Hemisphere summer (winter). This is due to field lines in the winter hemisphere moving much faster than those in the summer hemisphere.

- The solar generated Hall conductance causes the potential pattern to become asymmetric along the noonmidnight meridian, such that the dusk potential becomes larger in magnitude and the throat moves from 12:00 MLT toward dawn. The pressure distribution in the inner magnetosphere can be altered by $\pm 15 \%$.

- The potential during solar maximum is $\sim 11 \%$ smaller than the potential during solar minimum, when considering only the $f_{10.7}$ dependent conductance. This difference may decrease when the auroral conductances are included.

- The auroral precipitation can be emprically related to the field-aligned current through the use of an exponential function, where the constants are highly dependent on location and the sign of the field-aligned current.

- A constant polar cap conductance tends to remove some of the dawn-dusk asymmetry in the potential because it increases the constant conductance terms in the relationship between the currents and potential, thereby reducing the importance of the day-to-night gradient in the Hall conductance.

One strong caveat in this study is that the magnetosphere respresented within this paper is an MHD magnetosphere. While the MHD simulations correctly depict the basic shape of the pressure distribution in the magnetosphere, the amplitude is wrong. Within the MHD simulations, the pressure in the inner magnetosphere is typically underestimated by approximately an order of magnitude. For example, within the breaking region on the night-side, the MHD derived pressure is $\sim 1 \mathrm{nPa}$ (as shown in earlier figures within this paper), while observations (and ring-current modeling) of the pressure in this region are 5-100 $\mathrm{nPa}$ (Lui and Hamilton, 1992; Liemohn et al., 2001). This difference is due to the lack of energy discritization within the MHD codes (i.e. the codes lack the ability to model high energy particles) and they most likely underestimate the heating which occurs during the reconnection process in the tail. The study presented by Ridley et al. (2003) shows changes in the pressure distribution in the inner magnetosphere due to the thermosphere neutral winds, which were similar to those reported by Peymirat et al. (1998) and Peymirat et al. (2002), who use a more realistic model of the inner magnetospheric particles. Because the pressure changes were so similar, it is expected that the results found here are resprentative of the true magnetosphere.

Acknowledgements. This research was supported by NSF (grants ATM-9802149 and ATM-9980078) and the DoD MURI program (grant F4960-01-1-0359).

Topical Editor T. Pulkkinen thanks P. Janhunen for his help in evaluating this paper.

\section{References}

Ahn, B.-H., Robinson, R., Kamide, Y., and Akasofu, S.-I.: Electric conductivities, electric fields and auroral particle energy injection rate in the auroral ionosphere and their empirical relations to horizontal magnetic disturbances, Planet. Space Sci., 31, 641, 1983.

Ahn, B.-H., Richmond, A., Kamide, Y., Kroehl, H., Emery, B., de la Beaujardiére, O., and Akasofu, S.-I.: An ionospheric conductance model based on ground magnetic disturbance data, J. Geophys. Res., 103, 14,769, 1998.

Amm, O.: Comment on "A three-dimensional, iterative mapping procedure for the implementation of an ionospheremagnetosphere anisotropic Ohm's law boundary condition in global magnetohydrodynamic simulations", Ann. Geophysicae, 14, 773, 1996.

Brekke, A. and Moen, J.: Auroral ionospheric quiet summertime conductances, J. Atmos. Terr. Phys., 55, 1493, 1993.

Fedder, J. and Lyon, J.: The solar wind - magnetosphere - ionosphere current - voltage relationship, Geophys. Res. Lett., 14, 880, 1987.

Frahm, R., Winningham, J., Sharber, J. R., Link, R., Crowley, G., Gaines, E. E., Chenette, D. L., Anderson, B. J., and Potemra, T. A.: The diffuse aurora: A significant source of ionization in the middle atmosphere, J. Geophys. Res., 102, 28 203, 1997.

Fukushima, N.: Equivalence in ground geomagneitc effect of Chapman-Vestine's and Birkland-Alfvén's electric currentsystems for polar magnetic storms, Rep. Ionos. Space Res. Jpn., 23(3), 1969.

Fuller-Rowell, T. and Evans, D.: Height-integrated Pedersen and Hall conductivity patterns inferred from TIROS-NOAA satellite data, J. Geophys. Res., 92, 7606, 1987.

Galand, M., Fuller-Rowell, T., and Codrescu, M.: Response of the upper atmosphere to auroral protons, J. Geophys. Res., 106, 127, 2001.

Goodman, M. L.: A three-dimensional, iterative mapping procedure for the implementation of an ionosphere-magnetosphere anisotropic Ohm's law boundary condition in global magnetohydrodynamic simulations, Ann. Geophysicae, 13, 843, 1995.

Green, A., Lindenmayer, C., and Griggs, M.: Molecular absorption in planetary atmospheres, J. Geophys. Res., 69, 493, 1964.

Hardy, D., Gussenhoven, M., and Holeman, E.: A statistical model of auroral electron precipitation, J. Geophys. Res., 90, 4229, 1985.

Hardy, D., Gussenhoven, M., Raistrick, R., and McNeil, W.: Statistical and fuctional representation of the pattern of auroral energy flux, number flux, and conductivity, J. Geophys. Res., 92, 12275 , 1987.

Hill, T. and Rassbach, M.: Interplanetary magnetic field direction and the configuration of the dayside magnetosphere, J. Geophys Res., 80, 1, 1975. 
Iijima, T. and Potemra, T. A.: The amplitude distribution of fieldaligned currents at northern high latitudes observed by Triad, J. Geophys. Res., 81, 2165, 1976.

Knight, S.: Parallel electric fields, Planet. Space Sci., 21, 741, 1972.

Liemohn, M. W., Kozyra, J. U., Thomsen, M. F., Roeder, J. L., Lu, G., Borovsky, J. E., and Cayton, T. E.: The dominant role of the asymmetric ring current in producing the stormtime $D_{s t} *$, . Geophys. Res., 106, 10 883, 2001.

Lilensten, J., Blelly, P., Kofman, W., and Alcaydé, D.: Auroral ionospheric conductivities: a comparison between experiment and modeling, and theoretical $f_{10.7}$-dependent model for EISCAT and ESR, Ann. Geophysicae, 14, 1297, 1996.

Liou, K., Newell, P., Meng, C.-I., Brittnacher, M., and Parks, G.: Synoptic auroral distribution: A survey using Polar ultraviolet imagery, J. Geophys. Res., 102, 27 197, 1997.

Lui, A. and Hamilton, D.: Radial profiles of quiet time magnetospheric parameters, J. Geophys. Res., 97, 19325, 1992.

Lummerzheim, D., Rees, M. H., Craven, J., and Frank, L.: Ionospheric conductances derived from spectral auroral images, J. Atmos. Terr. Phys., 53, 281, 1991.

McHenry, M. and Clauer, C.: Modeled ground magnetic signitures of flux transfer events, J. Geophys. Res., 92, 11 231-11 240, 1987.

Moen, J. and Brekke, A.: The solar flux influence os quiet-time conductances in the auroral ionosphere, Geophys. Res. Lett., 20, 971, 1993.

Newell, P. and Meng, C.-I.: Mapping the dayside ionosphere to the magnetosphere according to particle precipitation characteristics, Geophys. Res. Lett., 19, 609, 1992.

Papitashvili, V., Rich, F., Heinemann, M., and Hairston, M.: Parameterization of the Defense Meterological Satellite Program ionospheric electrostatic potentials by the interplanetary magnetic field strength and direction, J. Geophys. Res., 104 (A1), 177-184, 1999 .

Peymirat, C., Richmond, A., Emery, B., and Roble, R.: A magnetosphere-thermosphere-ionosphere electrodynamics general circulation model, J. Geophys. Res., 103, $17467,1998$.

Peymirat, C., Richmond, A., and Roble, R.: Neutral wind influence on the electrodynamic coupling between the ionosphere and the magnetosphere, J. Geophys. Res., 107, 1029, 2002.

Powell, K., Roe, P., Linde, T., Gombosi, T., and Zeeuw, D. D.: A solution-adaptive upwind scheme for ideal magnetohydrodynamics, J. Comp. Phys., 154, 284, 1999.

Raeder, J., Berchem, J., and Ashour-Abdalla, M.: The importance of small scale processes in global MHD simulations: Some numerical experiments, in The Physics of Space Plasmas, edited by Chang, T. and Jasperse, J. R., vol. 14, p. 403, MIT Cent. for Theoret. Geo/Cosmo Plasma Phys., Cambridge, Mass., 1996.

Raeder, J., Berchem, J., and Ashour-Abdalla, M.: The Geospace Environment Modeling Grand Challenge: Results from a Global
Geospace Circulation Model, J. Geophys. Res., 103, 14787 , 1998.

Raeder, J., McPherron, R., Frank, L., Kokubun, S., Lu, G., Mukai, T., Paterson, W., Sigwarth, J., Singer, H., and Slavin, J.: Global simulation of the Geospace Environment Modeling substorm challenge event, J. Geophys. Res., 106, 281, 2001.

Richmond, A. and Kamide, Y.: Mapping Electrodynamic features of the high-latitude ionosphere from localized observations: Technique, J. Geophys. Res., 93, 5741, 1988.

Ridley, A., Crowley, G., Frahm, R., Winningham, D., Sharber, J., Russell III, J., and Roble, R.: Variations of the nitric oxide mass mixing ratio in the thermosphere as a function of $K_{p}$, Geophys. Res. Lett., 26, 1541, 1999.

Ridley, A., Zeeuw, D. D., Gombosi, T., and Powell, K.: Using steady-state MHD results to predict the global state of the magnetosphere-ionosphere system, J. Geophys. Res., 106, $30067,2001$.

Ridley, A., Hansen, K., Tóth, G., Zeeuw, D. D., Gombosi, T., and Powell, K.: University of Michigan MHD results of the GGCM Metrics challenge, J. Geophys. Res., 107(A10), 1290, doi:10.1029/2001JA000 253, 2002.

Ridley, A., Gombosi, T., Zeeuw, D. D., Clauer, C., and Richmond, A.: Ionospheric control of the magnetospheric configuration: Neutral winds, J. Geophys. Res., 108, 2002JA009 464, 2003.

Robinson, R., Vondrak, R., Miller, K., Dabbs, T., and Hardy, D.: On calculating ionospheric conductances from the flux and energy of precipitating electrons, J. Geophys. Res., 92, 2565, 1987.

Senior, C.: Solar and particle contributions to auroral heightintegrated conductivities from EISCAT data: a statistical study, Ann. Geophysicae, 9, 449, 1991.

Siscoe, G. L., Erickson, G., Sonnerup, B., Maynard, N., Schoendorf, J., Siebert, K., Weimer, D., White, W., and Wilson, G.: Hill model of transpolar potential saturation: Comparisons with MHD simulations, J. Geophys. Res., 107, 10.1029, 2002.

Solirelis, T. and Newell, P.: Boundary-oriented electron precipitation model, J. Geophys. Res., 105, 18 655, 2000.

Song, P., Gombosi, T., and Ridley, A.: Three-fluid Ohm's law, J. Geophys. Res., 106, 8149, 2001.

Spiro, R., Reiff, P., and Maher, J.: Precipitating electron energy flux and auroral zone conductances - An empirical model, J. Geophys. Res., 87, 8215, 1982.

Torr, D., Torr, M., Brinton, H., Brace, L., Spencer, N., Hedin, A., Hanson, W., Hoffman, J., Nier, A., Walker, J., and Rusch, D.: An experimental and theoretical study of the mean diurnal variation of $\mathrm{O}^{+}, \mathrm{NO}^{+}, \mathrm{O}_{2}^{+}$, and $\mathrm{N}_{2}^{+}$ions in the mid-latitude $F_{1}$ layer of the ionosphere, J. Geophys. Res., 84, 3360, 1979.

Weimer, D.: A flexible, IMF dependent model of high-latitude electric potential having "space weather" applications, Geophys. Res. Lett., 23, 2549, 1996. 